\title{
THE NUMERICAL MODELLING OF TIDES IN A SHALLOW SEMI- ENCLOSED BASIN BY A MODIFIED ELLIPTIC METHOD
}

by

\author{
Mulia M. SidjaBAT \\ (Manuscript received 1 April 1976)
}

\section{FOREWARD}

At the lime of Dr. Sidjabat's death in December 1974, he was involved in many projects and undoubtedly had plans for publishing many of his results. Unfortunately, most of his work was left in a form too sketchy to allow one to prepare it readily for publication with the exception of the present work. This work is based on Dr. Sidjabat's doctoral dissertation, finished in January 1970 at the University of Miami under the supervision of Russell Snyder, and thus had already been prepared for readers. I believe that Dr. Sidjabat had intended to publish some aspects of this work. The main editing job consisted in reducing the length to a convenient publishable size, and in making a few modifications and corrections. This job was not done as well as Dr. Sidjabat himself would have been able to do, and I apologize for and take responsibility for all shortcomings, of which there are undoubtedly many.

Russell Snyder has recently discovered errors in the computer programs used in this study. The main effects were to give values of the frictional coefficient $r$ which are too low by a factor of approximately $\sqrt{ } 2$, and to reduce the influence of the coriolis term. Thus, the conclusion that this term is unimportant in the Bight of Abaco may be overconfidently slated. Snyder also recalculated Sidjabat's Figure 9, giving the following result.

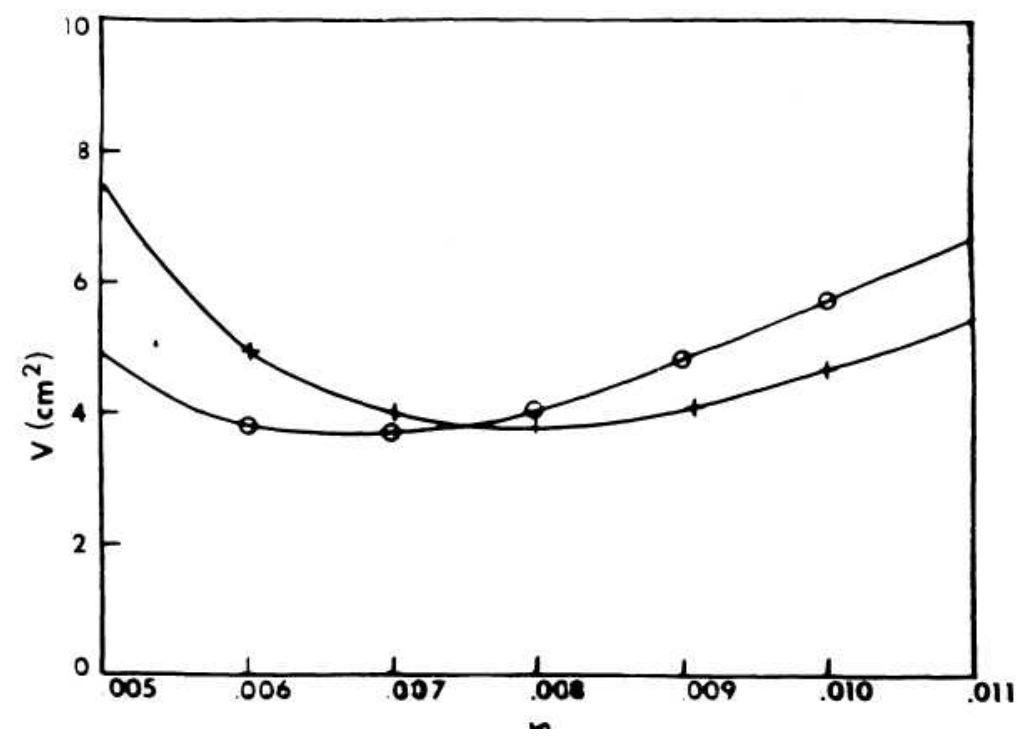


Here the crosses are for the actual Coriolis force and the zeroes are for the case $f=O$. Comparison of this figure with Sidjabat's Figure 9 shows that his r values are too low, and that the Coriolis force is important. Fortunately, Sidjabat's tidal distributions and other conclusions are not affected by those errors.

I am greatly indebted to Russell Snyder for his help and for furnishing the figures. This work was partially supported by the U.S. National Science Foundation through Grant GA-17137.

Gordon W. Groves

University of Hawail

April 1976

\begin{abstract}
A numerical model which renders possible the numerical solution of the nonlinear tidal equations by obtaining a solution for each individual tidal component is developed. For this purpose, a set of time independent non-linear equations for each tidal constituent is constructed. Each of these sets of equations is interrelated through the non-linear frictional terms, the approximation of which is accomplished by an iterative scheme. The method is tested for several models before it is applied to the real basin (Bight of Abaco). In order to evaluate the model and to construct the boundary conditions along the opening, a series of tidal observations were undertaken. The viability of the method is indicated by the fact that the results of computations using a coefficient of friction $\mathrm{r}=0.0034$ give good agreement with observations for all components and over all stations.
\end{abstract}

\title{
STATEMENT OF PROBLEM
}

The quantitative modeling of shallow water tides has long been of interest to oceanographers. A major consideration in this modeling has been 1) the representation of the frictional terms in the tidal equations and 2 ) the method of solution of the resulting equations. For physical reasons one expects the appropriate representation of the frictional terms to be non-linear. Frequently, a linear representation arising from a linearization of a quasi-quadratic non-linear representation has been assumed. This linearization can successfully be applied if the frictional force is small as in the case of a deep ocean. In shallow water, however, where the frictional force plays a significant role in the momentum balance (PR OU DM AN 1952; BOW DEN 1953), the linear theory has been inadequate. In particular it cannot describe several important aspects of shallow water tides, such as the interaction among tidal components and the associated generation of so-called shallow water tidal components. Non-linear theory gives deeper 


\section{MODELLING OF TIDES IN BASIN}

qualitative insight into the shallow water tide problem and permits the study of interaction phenomena, but the resulting equations are more difficult to solve.

Shallow water tidal computations in both model and real basins have been undertaken by various authors. In the beginning, these were accomplished by solving the linearized equations analytically. In order to obtain the solution in such a case, a harmonic form is assumed for the surface elevation and for the velocity and is introduced into the equations. Elimination of the velocity leads to an equation of elliptic type for the surface elevation. For basins of simple geometry and bottom topography, subject to uniform boundary conditions, an analytic solution for each tidal component can often be obtained and the tides can then be constructed by superimposing these individual solutions. In actuality, it is generally known that the bottom topography of a basin has a significant effect on the behavior of the tides. Thus, major difficulties arise if a real basin with more complicated depth distribution and more complex coastline has to be considered. Here, one has to resort to numerical analysis. This can be done by transforming the differential equations into a system of difference equations and solving these numerically with the aid of an electronic digital computer.

DEFANT (1919) was the first one to apply a numerical method to compute tides in canal-like seas, such as the Red Sea, where the linearized equations can be employed. His results are in good agreement with observation. In recent years, SGIBNEVA (1966) has applied an elliptic method to compute numerically the semidiurnal lunar tide $\mathrm{M}_{2}$ in a more complicated basin (Black Sea). His calculations are in satisfactory agreement with the measurements, but significant deviations are observed in shallow regions. These deviations apparently are due to the poor representation of the frictional force.

If a non-linear representation of friction is assumed, then different methods have to be developed. In order to have this type of problem well posed [in the sense of Hadamard (FORSYTHE \& WASOW 1964)], initial conditions are required in addition to boundary conditions. DRONKERS (1962) has demonstrated that for a pure harmonic tide one can reduce the tidal equations to time independent non-linear equations of elliptic type for the surface elevation. The present thesis essentially extends this technique to a compound tide. The Dronkers approximation permits one to eliminate the requirement for initial conditions, and attendant difficulties with the real characteristics, but apparently his method has not often been employed in tidal computations.

HANSEN (1962) has developed a technique of numerical modeling of tides by representing all derivatives of space and time variables by 
finite differences. A stepwise integration is performed starting from initial conditions until a steady state solution is obtained. By a special arrangement of the grid system used in modeling the area under consideration, Hansen's method can treat all the non-linearities in the equation without many problems. Hansen's method has been recently employed extensively to calculate tidal oscillations in various types of basins by a number of authors (GRIJALVA 1962; BRETTSCHNEIDER 1967), and their results agree quite well with observations. The disadvantage of Hansen's method is that it does not readily give information on the propagation of each individual component of the tide or on the nature of the interaction among the tidal constituents.

The purpose of the present study is to develop a model which renders possible the numerical solution of the non-linear tidal equations by obtaining a solution for each individual tidal component. This is accomplished in the following way. It is generally recognized that the tides can always be resolved in terms of the principal tidal components and their higher harmonics regardless of the complexity of the structure of the area or. the non-linearity of the motions. This resolution allows one to construct a set of time independent non-linear equations for each of the tidal constituents. However, each of these sets of equations is not totally independent from the others. They are inter-related through the non-linear frictional terms which are here taken to be quasi-quadratic in the velocity. An iterative method is employed to produce an approximation of the interaction terms. At each stage of the calculation, an elliptic type equation is obtained for each tidal component, which can be solved by a numerical method similar to that employed by Defant and Sgibneva.

The first step in investigating this approach is to design a basic elliptic method for a simple model basin, one of uniform depth and constant cross-sectional area, closed at one end with the other end open. One may then compare the numerical solution with the analytical solution obtained from simplified forms of the governing equations. The method is then tested on several idealized models before it is applied to a real basin. The convergence of the iterative scheme in approximating the interaction terms is investigated.

The method is then applied to a real semi-enclosed basin (Bight of Abaco). The tidal characteristics along the opening of the basin which are used as the boundary conditions for the numerical modeling, are determined from the experimental data by ordinary harmonic analysis. In order to check the validity of the proposed numerical model, these results are compared with the experimentally determined tidal topography of the area under study. This comparison allows the determination of an optimum value for the frictional coefficient. 


\section{Notation and Basic Equations}

A Cartesian coordinate system is employed throughout. This system is oriented so that the origin and the $x, y$ plane lie on the undisturbed water surface; $\mathrm{z}$ is positive upwards. Two dimensional horizontal vector quantities are capped with an arrow; two such quantities are the horizontal position vector:

$$
\vec{x} \equiv(x, y)
$$

and the vertically averaged horizontal velocity

$$
\vec{u} \equiv(u, v)
$$

The symbols that will be used are as follows:

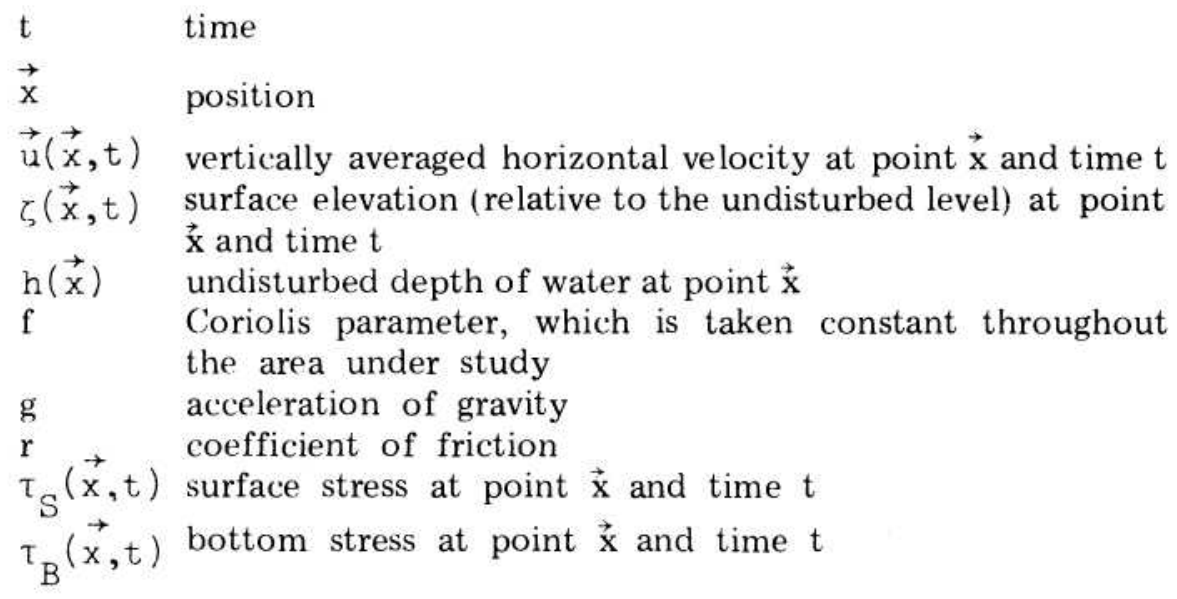

The basic equations which will be used in the analysis in this study result from the application of the physical principles of conservation of momentum and mass to the tidal process. This application results in one vector and one scalar partial differential equation with kinematic and dynamic boundary conditions at the surface and bottom (LAMB 1932, KINSMAN 1965). Neglect of several terms in the vertical component of the momentum equation namely the vertical acceleration of water particles and the vertical component of the Coriolis force, leads to the elimination of the pressure and its replacement by the hydrostatic 


\section{M. SIDJABAT}

approximation in the remaining equations. These equations are then vertically averaged to yield:

$$
\begin{gathered}
\frac{\partial}{\partial t} \vec{u}(\vec{x}, t)+\vec{u}(\vec{x}, t) \cdot \vec{\nabla} \vec{u}(\vec{x}, t)-\tau_{B}(\vec{x}, t)-\tau_{S}(\vec{x}, t)+ \\
f x \vec{u}(\vec{x}, t)+g \vec{\nabla} \zeta(\vec{x}, t)=0 \\
\frac{\partial}{\partial t} \zeta(\vec{x}, t)+\vec{\nabla} \cdot(h+\zeta(\vec{x}, t)) \vec{u}(\vec{x}, t)=0
\end{gathered}
$$

In the first of the above equations the notation $\mathrm{f} \mathrm{x} \mathrm{u}(\overrightarrow{\mathrm{x}}, \mathrm{t})$ denotes the horizontal vector quantity $(-\mathrm{fv}, \mathrm{fu})$. In the long wave approximation, the inertial terms are frequently neglected since their relative magnitude is small compared to the leading terms in ( la ) (although these terms may be significant in a shallow bay). The area under consideration is a small basin (wavelength is large compared to the length of the basin), so that direct tide generation by moon and sun is negligible. The tide is regarded as generated in the open ocean and propagated into the bay. The specification of a unique solution requires the application of proper boundary conditions (RICHTMYER \& MORTON 1967). The above equations are subject to two types of boundary conditions:

1. Fixed boundary (along the coast). The motion in the coastal region is fairly complicated and difficult to treat mathematically. The boundary condition here is determined by the nature of the bottom topography along the coast, which will be too complex if real topography is considered. An attempt will be made to assume a hypothetical vertical boundary and apply the condition

$$
\vec{n} \cdot \vec{u}(\vec{x}, t)=0
$$

where $\mathrm{n}$ is a unit horizontal vector perpendicular to the boundary. This assumption is permitted so long as the transport through the hypothetical boundary is insignificant (MIYASAKI 1964);

2. Free boundary (along the opening). The characteristic of the tides along the opening are given (i.e., determined from tidal observations),

$$
\zeta(\vec{x}, t)=g(\vec{x}, t)
$$




\section{MODELLING OF TIDES IN BASIN}

where $\mathrm{g}(\mathrm{x}, \mathrm{t})$ is a well behaved function.

Some consideration of the physical characteristics of the tides in shallow water encompassed by the above equations will be helpful. The tides of a basin are considered to be part of a co-oscillating system (DEFANT 1961) in which the period is determined by the tides in the outer sea. The character of the motion in the basin is influenced qualitatively by the geometry of the basin in several ways:

1. If the estuary is convergent, the amplitude tends to increase.

2. Energy dissipation by bottom friction tends to reduce the amplitude of the incident wave.

3. If the basin is small, the effect of the Coriolis force may be negligible.

Since the region under study is a shallow basin, the resistance force has an important effect, so that special care must be taken in evaluating the influence of this force. The time lag between the tide in the bay and that along the opening is a prominent manifestation of this property. BOW DEN (1953) pointed out that in a shallow bay, the frictional effect is more important than the Coriolis force to -the tidal motion.

\section{Discussion of the Frictional Terms}

The physical nature of bottom stress due to friction is difficult to ascertain. It has been customary to assume that this stress $\tau_{\mathrm{B}}$, is approximated by the formula

$$
\vec{i}_{B}=-\frac{r}{h}|\vec{u}| \vec{u}
$$

where $u$ is the mean current (PROUDMAN 1952, BOWDEN 1953). This "quasi-quadratic" formulation makes the friction due to the bottom proportional to the square of speed and in a direction opposite to that of the flow.

Due to the intractability of the non-linear formulation, various attempts have been made to approximate the problem. Frequently, a linearized form of the friction is employed. Several alternate forms have been used, but. they are still essentially linearizations. BOWDEN (1953) and IPPEN (1966), for example, approximated the frictional coefficient from the mean velocity throughout the bay, a method which can only be applied to a basin whose boundary can be represented by 
a simple function. Some difficulties arise from this linearization. If one replaces $r|u| u$ by the expression $R u$, then the value of $R$ depends upon the velocity $u$. The value of $R$ here will be different from the value of $r$ and it has a dimension (time $)^{-1}$. The other difficulty is that the interaction among the tidal components is absent. The linearized anlysis of tidal motion can lead to results which are reasonably accurate only when the frictional force is small, such as in the case of deep water.

When a tide propagates into a shallow basin, its motion is greatly distorted due to the bottom friction and shallow water constituents are generated. These constituents can only be generated by a non-linear frictional term and by other non-linearities in Eqs. ( la ) and ( $1 \mathrm{~b}$ ). The non-linear terms determine the mutual interaction of the tidal constituents and the propagation and shape of tides in shallow water.

The friction law requires the use of a friction coefficient, $r$, the numerical value of which depends upon the physical state of the medium as well as upon the nature of the bottom of the sea. A value of $r=0.003$ has been found by actual observations of several workers (BOWDEN \& FAIRBAIRN 1952, ROSSITER 1963) to be of the right order of magnitude for tidal currents. However, in practice, the value of $r$ used for a particular area should be adjusted to compare with actual observations.

\section{Discussion of Coriolis Term}

It has been generally stated that for a relatively narrow basin, the Coriolis force can be neglected (PROUDMAN 1952, DEFANT 1961). However, DRONKERS (1962) suggested that in a shallow bay with a wide opening, it may be important to consider the effect of this force on the tidal motion. In order to evaluate this question, one should consider the relative magnitude of the Coriolis force compared to other terms in the governing equations.

For the region under consideration it can be easily shown, that the non-linear acceleration is much smaller than friction term and that the ratio of the Coriolis term to the pressure gradient is approximately 0.2 . This shows that the magnitude of the Coriolis force is substantially smaller than the leading terms. In the actual situation the relative magnitudes may differ from the ratio obtained above. Since the inclusion of this term into a numerical model will not introduce a major problem, it is just as well that it be taken into account. In doing this, one will be able to determine the relative importance of the Coriolis term. 


\section{METHOD OF ANALYSIS}

In order to transform the tidal equations into an elliptic type equation for the surface elevation, one has to transform a set of nonlinear time dependent equations into several coupled sets of nonlinear time independent equations. This can be done by representing the surface elevation and current as harmonic functions of time and substituting into the governing equations. However, the resulting time-independent equations are not independent from each other. Each set contains a nonlinear term representing self interaction and interaction with other tidal components.

The harmonic representation of the surface elevation and current are as follows

$$
\begin{aligned}
& \zeta(\vec{x}, t)=\sum_{n} \zeta_{n}(\vec{x}) e^{i \omega n t}+\zeta^{\prime} \\
& \vec{u}(\vec{x}, t)=\sum_{n} \vec{U}_{n}(\vec{x}) e^{i_{\omega n} t}+\vec{U}^{\prime}
\end{aligned}
$$

where the summation extends over both positive and negative values of the index $\mathrm{n} ; \zeta_{\mathrm{n}}(\mathrm{x})$ and $\mathrm{U}_{\mathrm{n}}(\mathrm{x})$ are complex functions of position and satisfy the relation

$$
\begin{aligned}
& \zeta_{n}(\vec{x})=\zeta_{-n}^{\star}(\vec{x}) \\
& \vec{U}_{n}(\vec{x})=\vec{U}_{-n}^{\star}(\vec{x})
\end{aligned}
$$

where denotes complex conjugate. The relation

$$
\omega_{n}=-\omega_{-n}
$$

holds for each tidal frequency $\omega_{\mathrm{n}}$, and the sum ranges over all principal tidal frequencies and linear combinations there of; $f^{\prime}$ and $U^{\prime}$ are the residual surface elevation and current. These are random terms induced by the surface stress $\tau_{\mathrm{S}}$.

By substituting the expression ( 4 ) and ( 5 ) into the tidal equations ( la ) and ( $1 \mathrm{~b})$, the following equations are obtained: 


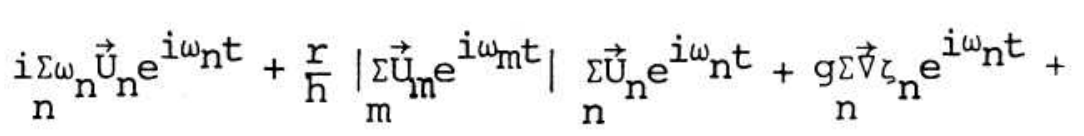

$$
\begin{aligned}
& \text { random terms }=0 \\
& { }_{n}^{i \sum \omega_{n} \zeta n} e^{i \omega_{n} t}+\underset{n}{\Sigma \vec{\nabla}} \cdot\left(\vec{H}_{n} e^{i \omega_{n} t}\right)+\text { random terms }=0
\end{aligned}
$$

For simplicity of the notation, the $\mathrm{x}$ dependence of the variables $\zeta_{\mathrm{n}}(\mathrm{x})$ and $\mathrm{U}_{\mathrm{n}}(\mathrm{x})$ has been suppressed. The non-linear acceleration and Coriolis terms, for the time being, are not included. In addition, the surface elevation $\zeta_{n}$ is regarded as small compared to the depth, which enables one to linearize the continuity equations. Also, it is assumed that the tidal component of the wind stress is negligible. Therefore, this stress contributes only to random terms in the above equations.

In order to construct the set of equations for each tidal component, further analysis of the frictional term is necessary. The frictional term in Eq. ( 6a ) can be expressed as

$$
\vec{\tau}_{B}=-\frac{r}{h} \lambda\left(1+\frac{1}{\lambda^{2}} \sum_{n m} \sum_{n} \vec{U}_{n} \cdot \vec{U}_{m} e^{i\left(\omega_{n}+\omega_{m}\right) t}\right)_{1}^{\frac{1}{2}} \sum_{1} \vec{U}_{1} e^{i \omega_{1} t}
$$

where $\lambda$ be defined by

$$
\lambda^{2}(\vec{x})=\sum_{n} \vec{U}_{n} \cdot \vec{U}_{-n}
$$

The square root can be expanded in a Taylor series, e.g.,

$$
\begin{aligned}
& \vec{\tau}_{B}=-\frac{T}{h} \lambda\left(1+\frac{1}{2 \lambda^{2}} \sum_{m} \sum_{n} \vec{U}_{m} \cdot \vec{U}_{n} e^{i\left(\omega_{m}+\omega_{n}\right) t}+\right. \\
& m \neq-n
\end{aligned}
$$

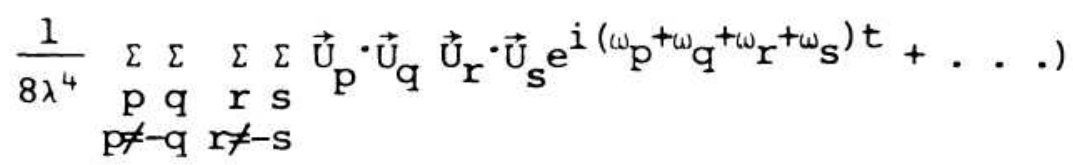


MODELLING OF TIDES IN BASIN

For simplicity let

$$
\vec{F}_{B}=-\frac{r}{h} \lambda\left(T_{0}+T_{1}+T_{2}+. . .\right) \sum_{1} \vec{U}_{1} e^{i \omega_{1} t}(7)
$$

where $T_{O}, T^{\wedge}$ and $T 2$ are the terms that contain the zero, first and second order term with respect to e of the Taylor Series, where $e$ is given by

$$
\varepsilon=\frac{1}{\lambda^{2}} \sum_{n m} \sum \vec{U}_{n} \cdot \vec{U}_{m} e^{-\left(\omega_{n}+\omega_{m}\right) t}
$$

One can clearly see that the zero order term will contribute to the set of equations for a principal tidal component. Most of the terms generated by $\tau_{1}$ will not contribute because the sum of $\omega_{\mathrm{m}}$ and $\omega_{n}$ will never be zero (note that $m \neq-n$ ). However, certain combinations of $\omega_{m}, \omega_{n}$ and $\omega_{1}$ may produce a frequency equal to a principal frequency, e.g.,

$$
\begin{aligned}
& m=n \\
& 1=-n
\end{aligned}
$$

In a basin where $\mathrm{M}_{2}$ is the dominant tide (as in the Bight of Abaco) $\lambda$ is determined primarily by the $\mathrm{M}_{2}$ fields. It follows that $T_{1}$ can produce a significant contribution only for the $\mathrm{M}_{2}$ set of equations. Investigation of this case suggests that the size of this contribution is less than $25 \%$ of the contribution from $T_{O}$. As the principal objective is to demonstrate the viability of a modified elliptic method to model tides in a shallow basin and not to investigate all the ramifications of the assumption of quasiquadratic resistance, $\mathrm{T}_{1}$ has been neglected in the computations. It should be pointed out, however, that $T_{1}$ could be included in the computation within the framework of the present method of analysis.

As in the case of $T_{1}$, most of the terms of $T_{2}$ will not generate principal tidal frequencies, except for $\mathrm{p}=-\mathrm{r}$ and $\mathrm{q}=-\mathrm{s}$, e.g.,

$$
\begin{aligned}
\mathrm{T}_{2}{ }^{\prime}=\frac{1}{8 \lambda^{4}} & \sum_{p} \sum_{\mathrm{q}} \overrightarrow{\mathrm{U}}_{\mathrm{p}} \cdot \overrightarrow{\mathrm{U}}_{-\mathrm{q}} \overrightarrow{\mathrm{U}}_{-\mathrm{p}} \cdot \overrightarrow{\mathrm{U}}_{\mathrm{q}} \\
\mathrm{p} & \neq-\mathrm{q}
\end{aligned}
$$

the constant part of $\mathrm{T}_{2}$, can be written as 


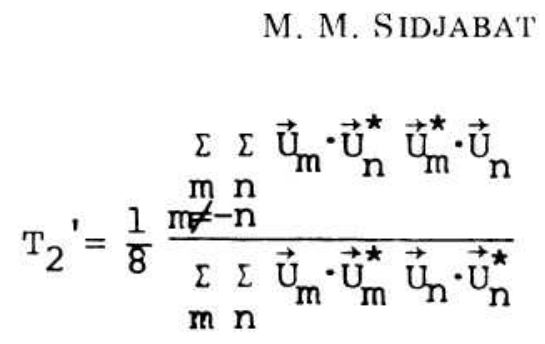

The following notation is introduced in order to carry out further reduction of this expression,

$$
\begin{aligned}
& {\left[\vec{U}_{n}\right]: } \text { is the amplitude of } \vec{U}_{n}, e \cdot g \cdot \text {, if } \\
& \vec{U}_{n}=\left(U_{r}+i U_{i m}, v_{r}+i v_{i m}\right) \text { then } \\
& {\left[\vec{U}_{n}\right] \equiv\left[\left(U_{r}^{2}+U_{i m}^{2}\right)^{\frac{1}{2}},\left(v_{r}^{2}+v_{i m}^{2}\right)^{\frac{1}{2}}\right] } \\
&\left|\vec{U}_{n}\right|: \text { is the magnitude of } \vec{U}_{n}, e \cdot g \cdot, \\
&\left|\vec{U}_{n}\right|=\left(\vec{U}_{n} \cdot \vec{U}_{n}\right)^{\frac{1}{2}}
\end{aligned}
$$

Note that $\left[\vec{U}_{n}\right]$ is a real vector quantity and $\overrightarrow{\mathrm{U}}_{n}$ is a complex scalar quantity. Then it can be demonstrated that

$$
\begin{gathered}
{\left[\overrightarrow{\mathrm{U}}_{n}\right]=\left[\overrightarrow{\mathrm{O}}_{n}^{\star}\right]} \\
\left|\left[\overrightarrow{\mathrm{U}}_{n}\right]\right|=\left(\overrightarrow{\mathrm{U}}_{n} \cdot \overrightarrow{\mathrm{U}}_{\mathrm{n}}^{\star}\right)^{\frac{3}{2}}
\end{gathered}
$$

and

$$
\left[\overrightarrow{\mathrm{U}}_{\mathrm{m}} \cdot \dot{\mathrm{U}}_{\mathrm{n}}^{\star}\right]^{2}=\dot{\mathrm{U}}_{\mathrm{m}} \cdot \dot{\mathrm{U}}_{\mathrm{n}}^{\star} \dot{\mathrm{U}}_{\mathrm{m}} \cdot \dot{\mathrm{U}}_{\mathrm{n}}
$$

$\left(\left[\vec{U}_{\mathrm{m}} \cdot \overrightarrow{\mathrm{U}}_{\mathrm{n}}^{*}\right]\right)$ denotes the amplitude of the complex scalar quantity $\left(\dot{\mathrm{U}}_{\mathrm{m}} \cdot \overrightarrow{\mathrm{U}}_{\mathrm{n}}^{*}\right.$ ). Consequently, $\mathrm{T}_{2}$ ' is given by

$$
\mathrm{T}_{2}^{\prime}=\frac{1}{8} \frac{\sum_{\mathrm{n}}\left(\left[\overrightarrow{\mathrm{u}}_{\mathrm{m}} \cdot \overrightarrow{\mathrm{U}}_{\mathrm{n}}\right]\right)^{2}-}{\sum_{\mathrm{m} \mathrm{n}}\left(\left|\left[\dot{\mathrm{U}}_{\mathrm{m}}\right]\right|\left|\left[\overrightarrow{\mathrm{U}}_{\mathrm{n}}\right]\right|\right)^{\prime}}
$$


Note that the restriction for $n \neq-m$ for the sum in the numerator is cancelled, and a positive number, $\delta$, is introduced. Applying the Schwarz inequality for complex functions, one has

$$
\begin{aligned}
{\left[\dot{\mathrm{U}}_{\mathrm{m}} \cdot \dot{\mathrm{U}}_{\mathrm{n}}^{\star}\right] } & \leq\left[\overrightarrow{\mathrm{U}}_{\mathrm{m}}\right]\left[\overrightarrow{\mathrm{U}}_{\mathrm{n}}\right] \\
& \leq\left|\left[\overrightarrow{\mathrm{U}}_{\mathrm{n}}\right]\right| \mid\left[\overrightarrow{\mathrm{U}}_{\mathrm{m}}\right] ! \cos n_{\mathrm{m}}
\end{aligned}
$$

Where $0_{\mathrm{mn}}$ is the angle (real number) between the real vector $U_{\mathrm{n}}$ and $\mathrm{U}_{\mathrm{m}}$. Therefore, it is seen that the numerator of ( 8 ) is less than the denominator, which implies that

$$
\mathrm{T}_{2}{ }^{\prime} \div \frac{1}{8}
$$

In a similar manner, the higher order terms of ( 7 ) can presumably he demonstrated to be smaller than the second term. Therefore, to a good approximation, the frictional term is represented by

$$
v_{B}=-\frac{r}{h}, \sum_{n} \vec{U}_{n} e^{i \omega_{n} t}
$$

In practical applications, one of the terms of the tidal expansion very often predominates over the others, which enables one to represent the frictional force by a single term that has the frequency of the principal component, BOWDEN (1953) and ORONKERS (1962) have approximated the non-linear frictional terms for a pure harmonic tide by expanding these terms in a Fourier series in time. The present scheme is essentially a generalization of this expansion to a multi-component almost periodic tide.

Now, the set of equations for a particular frequency which is obtained from above is

$$
\begin{aligned}
& 1 \omega_{n} \dot{\mathrm{U}}_{\mathrm{n}}+\frac{r}{\mathrm{~h}} \times \overrightarrow{\mathrm{U}}_{\mathrm{n}}+\overrightarrow{\mathrm{g}}_{\mathrm{n}}=0 \\
& i w_{n} \zeta_{n}+\vec{v} \cdot\left(h \vec{U}_{n}\right)=0
\end{aligned}
$$

Because $\lambda(x)$ depends upon all the $U_{n}(x)$, the above set is both nonlinear and coupled to the other harmonic sets. On the other hand, if $\lambda(\mathrm{x})$ is treated as a known function of position the set is linear and 


\section{M. SIDJABAT}

uncoupled. In this case, the solution for $\zeta_{\mathrm{n}}(\mathrm{X})$ and $\mathrm{U}_{\mathrm{n}}(\mathrm{x})$ can be obtained with comparative ease. This situation suggests the following iterative procedure for finding an approximate solution to the problem. Let the trial solution $\zeta^{\circ}(\mathrm{x}), \mathrm{Un}(\mathrm{x})$ satisfy the frictionless system of equations

$$
\begin{array}{r}
i \omega_{n} \dot{U}_{n}^{(0)}+g \nabla \zeta_{n}^{(0)}=0 \\
i u_{n} \zeta_{n}^{(0)}+\nabla \cdot\left(h \vec{U}_{n}^{(0)}\right)=0
\end{array}
$$

and let

$$
\begin{gathered}
i \omega_{n} \vec{U}_{n}^{(i+1)}+\frac{r}{h} \lambda^{i} \vec{U}_{n}^{(i+1)}+g \vec{\nabla} \zeta_{n}^{(i+1)}=0 \\
i \omega_{n} \zeta_{n}^{(i+1)}+\nabla \cdot\left(h \vec{U}_{n}^{(i+1)}\right)=0, \\
i=0,1,2, \ldots
\end{gathered}
$$

At each state of the calculation, $\mathrm{U}_{\mathrm{n}}$ can be eliminated to give an elliptic type equation of the form

$$
A_{n} \frac{\partial^{2}}{\partial x^{2}} \zeta_{n}+B_{n} \frac{\partial^{2}}{\partial y^{2}} \zeta_{n}+C_{n} \frac{\partial}{\partial x} \zeta_{n}+D_{n} \frac{\partial}{\partial y} \zeta_{n}+E_{n} \zeta_{n}=F_{n}
$$

where $A_{n}, B_{n}, C_{n}, D_{n}, E_{n}$ and $F_{n}$ are coefficients which can be computed for each $\mathrm{x}$. Applying the boundary conditions ( 2 ) with appropriate substitution from ( $6 \mathrm{~b}$ ), the above equation becomes subject to the boundary condition

$$
\vec{\nabla}_{n} \cdot \zeta=0
$$

which makes the problem well posed of Neumann type (FORYSTHE \& WASOW 1960).

The approximation of a non-linear acceleration term can be performed in a similar way to that used in the approximation of the factional term. Consider the inertial term 


$$
(\vec{u} \cdot \nabla)=\left(\sum_{n} \vec{U}_{n} e^{i \omega_{n} t} \cdot \vec{\nabla}\right) \sum_{m} \vec{U}_{m} e^{i \omega_{m} t}
$$

and let the solution of the tidal equations for a component of frequency $\omega_{\mathrm{k}}$ be sought. Then the terms that would contribute from the above expression are the ones the sum of whose frequencies are $\omega_{\mathrm{k}}$, e.g.,

$$
w_{m}+w_{n}=w_{k}
$$

This relation is satisfied when

$$
\begin{aligned}
& \omega_{n}=w_{K} \\
& \omega_{m}=w_{O_{1}} \\
& \omega_{K}=\omega_{M_{2}}
\end{aligned}
$$

where $\omega_{K_{1}}, \omega_{O_{1}}$ and $\omega_{M_{2}}$ are the frequencies of $K_{1}, O_{1}$ and $M_{2}$ tidal components respectively. However, this term can be neglected because its magnitude is small. In Hansen's method, the effect of this inertial term is not noticeable; it is included in his calculation only for the sake of stability of the computation.

The Coriolis term can also be included in the computations by evaluating it at $i^{\text {th }}$ level of the computation. This can be done because the relative magnitude of the Coriolis term is small compared to the other terms in the momentum equation.

To summarize the above discussion, the scheme of computation can be formulated as follows. First, the equations are linearized by setting $\lambda^{(0)}=0$, then solved to find the approximate $\zeta_{1}{ }^{(1)}, \zeta_{2}{ }^{(1)} \ldots$ and $\vec{U}_{1}{ }^{(1)}, \vec{U}_{2}{ }^{(1)}$. . . . From these solutions, $\lambda^{i}(\vec{x})$ is computed. The velocities are also used to evaluate the new Coriolis term for the next stage of computation. By solving the new equations, new and better approximations of the $\zeta_{n}$ and $\vec{U}_{n}$ are generated. This iterative procedure is continued until convergence is achieved to within a prescribed tolerance.

In dealing with the iterative procedure, it is important to know whether the computation yields the solution of the problem. As is commonly the case, the iterative scheme results in the construction of a sequence of numbers, and one wishes to know the condition for convergence of this sequence. The mathematical answer to these questions 


\section{M. SIDJABAT}

is beyond the scope of this paper. However, the author is inclined to judge the performance of the iterative scheme used here in a purely pragmatic way. The method was tried out in a number of models, and it worked satisfactorily.

\section{NUMERICAL MODEL}

For the numerical solution of boundary value problems, it is common to replace the differential equation by an approximate difference equation, and the continuous region in which the solution is desired by a set of discrete points. For this purpose, the entire region under consideration is divided into a network of equal squares of side L (Fig. la). This permits one to reduce the differential equations to a system of algebraic equations which involves many unknowns.

As has been mentioned previously, the frictional term is approximated from the previous velocity field. Then for given tidal component Eq. (1) can be written as follows

$$
\begin{aligned}
i \omega U+\frac{r}{h} \lambda^{\prime} U+g \frac{\partial \zeta}{\partial x} & =f V^{\prime} \\
i \omega V+\frac{r}{h} \lambda^{\prime} V+g \frac{\partial \zeta}{\partial y} & =-f U^{\prime} \\
i \omega \zeta+\frac{\partial h U}{\partial x}+\frac{\partial h V}{\partial y} & =0
\end{aligned}
$$

where' denotes the previous field. Note that the Coriolis force is also evaluated from the previous velocity field in order to simplify the numerical calculation. Then, $\lambda^{\prime}, \mathrm{U}^{\prime}$ and $\mathrm{V}^{\prime}$ are known functions of $\mathrm{x}$ and $\mathrm{y}$. From these equations, one can express $\mathrm{U}$ and $\mathrm{V}$ in terms of $\lambda^{\prime}, \mathrm{U}^{\prime}, \mathrm{V}^{\prime}$ and $\zeta$

$$
\begin{aligned}
& U=\frac{g\left(i \omega h^{\prime}+h r \lambda^{\prime}\right) \frac{o \zeta}{\partial x}}{h^{2} \omega^{2}+r^{2} \lambda^{\prime 2}}-\frac{f V^{\prime}\left(i \omega h^{2}+h r \lambda^{\prime}\right)}{h^{2} \omega^{2}+r^{2} \lambda^{\prime 2}} \\
& V=\frac{g\left(i \omega h^{2}+h r \lambda^{\prime}\right) \frac{\partial \zeta}{\partial y}}{h^{2} \omega^{2}+r^{2} \lambda^{\prime 2}}+\frac{f U^{\prime}\left(i \omega h^{2}+h r \lambda\right)}{h^{2} \omega^{2}+r^{2} \lambda^{\prime 2}}
\end{aligned}
$$

Substituting (11a) and (11b) into (10c), a single second order elliptic type equation for the complex surface elevation is obtained. 
MODELLING OF TIDES IN BASIN

$$
\begin{gathered}
G \nabla^{2} \zeta+i F \nabla^{2} \zeta+\frac{\partial G}{\partial x} \frac{\partial \zeta}{\partial x}+\frac{\partial G}{\partial y} \frac{\partial \zeta}{\partial y}+ \\
i\left(\frac{\partial F}{\partial x} \frac{\partial \zeta}{\partial x}+\frac{\partial F}{\partial y} \frac{\partial \zeta}{\partial y}\right)+i \omega \zeta=0
\end{gathered}
$$

where $F, G$ and $Q$ are defined by

$$
\begin{aligned}
& F=\frac{g \omega h^{2}}{\omega^{2} h^{2}+r^{2} \lambda^{\prime 2}} \\
& G=\frac{g r \lambda^{\prime} h}{\omega^{2} h^{2}+r^{2} \lambda^{\prime 2}} \\
& Q=\frac{\partial}{\partial x} \frac{h f\left(i \omega h^{2}+r h \lambda^{\prime}\right) V^{\prime}}{h^{2} \omega^{2}+r^{2} \lambda^{\prime 2}}+\frac{\partial}{\partial y} \frac{h f\left(i \omega h^{2}+r \lambda^{\prime} h\right) U^{\prime}}{h^{2} \omega^{2}+r^{2} \lambda^{\prime 2}}
\end{aligned}
$$

Now, let $U, V$ and $\zeta$ be represented in terms of their real and imaginary parts, e.g.

$$
\begin{aligned}
& \mathrm{U}=\mathrm{U}_{\text {re }}+i U_{i m} \\
& \mathrm{v}=\mathrm{v}_{\text {re }}+i v_{i m} \\
& \zeta=\zeta_{\text {re }}+i \zeta_{\mathrm{im}}
\end{aligned}
$$

Substituting these expressions into (12) and separating the real and imaginary parts, two linear elliptic equations are obtained,

real part

$$
\begin{gathered}
G \nabla^{2} \zeta_{r e}+\frac{\partial G}{\partial x} \frac{\partial \zeta_{r e}}{\partial x}+\frac{\partial G}{\partial y} \frac{\partial \zeta_{\text {re }}}{\partial y}-F \nabla^{2} \zeta- \\
\frac{\partial F}{\partial x}-\frac{\partial \zeta_{i m}}{\partial x}+\frac{\partial F}{\partial y} \frac{\partial \zeta_{i m}}{\partial y}-\omega \zeta_{i m}=Q_{\text {re }}
\end{gathered}
$$


imaginary part

$$
\begin{gathered}
G \nabla^{2} \zeta_{i m}+\frac{\partial G}{\partial x} \frac{\partial \zeta_{i m}}{\partial x}+\frac{\partial G}{\partial y} \frac{\partial \zeta_{i m}}{\partial y}+F \nabla^{2} \zeta_{r e}+ \\
\frac{\partial F}{\partial x} \frac{\partial \zeta_{r e}}{\partial x}+\frac{\partial F}{\partial y} \frac{\partial \zeta_{r e}}{\partial y}+\omega \zeta_{\text {im }}=Q_{i m}
\end{gathered}
$$

The boundary condition along the hypothetical coast parallel to the $\mathrm{x}$-axis is obtained by setting $\mathrm{V}=0$, from which the following equations result

$$
\begin{aligned}
& g \frac{\partial \zeta_{\text {re }}}{\partial y}+f U_{r e}^{\prime}=0 \\
& g \frac{\partial \zeta_{\text {im }}}{\partial y}+f U_{i m}^{\prime}=0
\end{aligned}
$$

In a completely analogous manner, the boundary condition along the coast parallel to the $y$-axis are

$$
\begin{aligned}
& g \frac{\partial \zeta_{r e}}{\partial x}-f v_{r e}^{\prime}=0 \\
& g \frac{\partial \zeta_{i m}}{\partial x}-f V_{i m}^{\prime}=0
\end{aligned}
$$

A central difference approximation is used in compiling the algorithm of the problem, e.g.,

$$
\begin{aligned}
\frac{\partial \zeta}{\partial x} & =\frac{\zeta(E)-\zeta(W)}{2 L} \\
\frac{\partial \zeta}{\partial x^{2}} & =\frac{\zeta(E)+\zeta(W)-2 \zeta(C)}{L^{2}} \\
\nabla^{2} \zeta & =\frac{\zeta(E)+\zeta(W)+\zeta(N)+\zeta(S)-4 \zeta(C)}{L^{2}}
\end{aligned}
$$


MODELLING OF TIDES IN BASIN

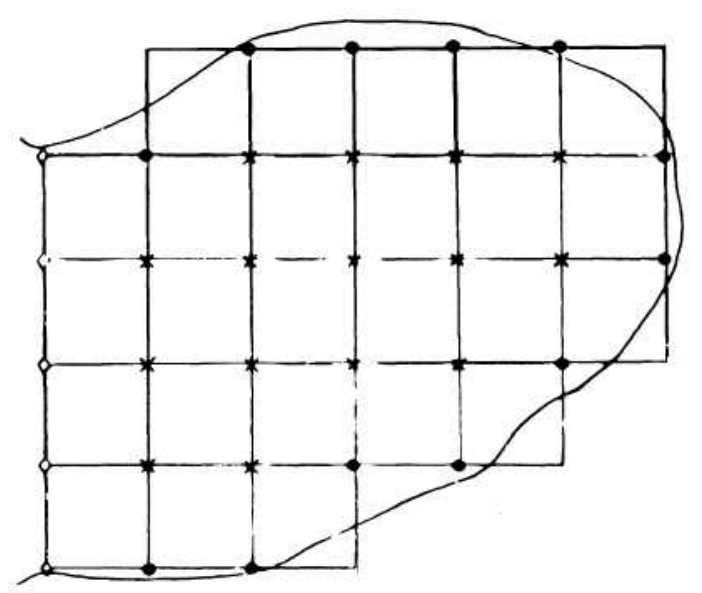

(a)

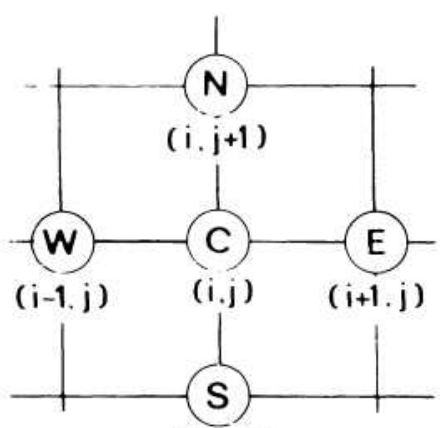

(i. j-1)

(b)

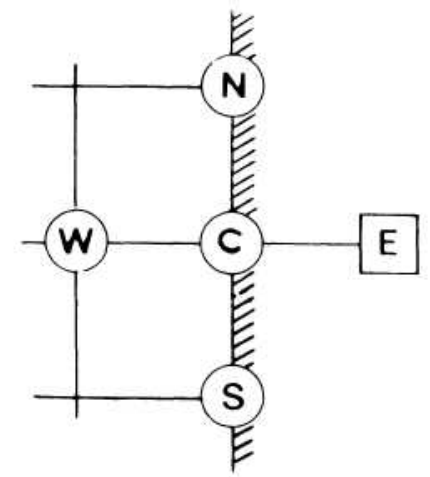

(C)

Pigure 1. Grid points for an arbitrary region (a) and coding systems used in the numerical model ( $b$ and e). The symbols used in this figure are defined as follows :
$\widehat{\Upsilon}$ opening point
$\therefore$ interior point
- boundary point
i! fictitious point 


\section{M. SIDJABAT}

where the notation of E, VV, N, S and C stand for East, West, North, South and Central points, respectively (Fig. lb).

Then, Eq. (13) can be written in finite difference form in the following manner

$$
\begin{aligned}
& \frac{G(C)}{\omega L^{2}}\left(\zeta_{r e}(W)+\zeta_{r e}(E)+\zeta_{r e}(N)+\zeta_{r e}(S)-4 \zeta_{r e}(C)\right)+ \\
& \frac{G x}{2 \omega L}\left(\zeta_{r e}(E)-\zeta_{r e}(W)\right)+\frac{G_{y}}{2 \omega L}\left(\zeta_{r e}(N)-\zeta_{r e}(S)\right)+ \\
& \left.\frac{F(C)}{\omega L^{2}}{ }^{\left(\zeta_{i m}\right.}(W)+\zeta_{i m}(E)+\zeta_{i m}(N)+\zeta_{i m}(S)-4 \zeta_{i m}(C)\right)- \\
& \frac{F_{x}}{2 \omega L}\left(_{i m}(E)-\zeta_{i m}(W)\right)-\frac{F_{y}}{2 \omega L}\left(\zeta_{i m}(N)-\zeta_{i m}(S)\right)- \\
& \omega \zeta_{i m}(C)=\Omega_{r e}(C)
\end{aligned}
$$

and Eq. (14) becomes

$$
\begin{aligned}
& \frac{G(C)}{\omega L^{2}}\left(\zeta_{i m}(W)+\zeta_{i m}(E)+\zeta_{i m}(N)+\zeta_{i m}(S)-4 \tau_{i m}(C)\right)+ \\
& \frac{G x}{2 \omega L}\left(\zeta_{i m}(E)-\zeta_{i m}(W)\right)+\frac{G y}{2 \omega L}\left(\zeta_{i m}(N)-\zeta_{i m}(S)\right)+ \\
& \frac{F(C)}{\omega L^{2}}\left(\zeta_{r e}(W)+\zeta_{r e}(E)+\zeta_{r e}(N)+\zeta_{r e}(S)-4 \zeta_{r e}(C)\right)+ \\
& \frac{F_{X}}{2 \omega L}\left(\zeta_{r e}(E)-\zeta_{r e}(W)\right)+\frac{F_{y}}{2 \omega L}\left(\zeta_{r e}(N)-\zeta_{r e}(S)\right)- \\
& \omega \zeta_{r e}(C)=Q_{i m}(C)
\end{aligned}
$$


where $F_{x}, F_{y}, G_{x}$ and $G_{y}$ are given by

$$
\begin{aligned}
& F_{X}=\frac{F(E)-F(W)}{2 L} \\
& F_{y}=\frac{F(N)-F(S)}{2 L} \\
& G_{X}=\frac{G(E)-G(W)}{2 L} \\
& G_{y}=\frac{G(N)-G(S)}{2 L}
\end{aligned}
$$

For the points which are located on the boundaries, a central difference approximation cannot be employed, unless so-called fictitious boundary points are introduced (Fig. 1c). By so doing, one is able to set the relation between the fictitious points and the interior points by using the boundary conditions $(15 \mathrm{a}, \mathrm{b})$ or $(16 \mathrm{a}, \mathrm{b})$ at the boundary points. To obtain more insight into this, consider a point on the east boundary. The boundary conditions in a finite difference form are

$$
\begin{aligned}
& g \frac{\bar{\zeta}_{r e}(E)-\zeta_{r e}(W)}{2 L}+f U_{r e}^{\prime}(C)=0 \\
& g \frac{\bar{\zeta}_{i m}(E)-\zeta_{i m}(W)}{2 L}+f U_{i m}^{\prime}(C)=0
\end{aligned}
$$

where an overbar denotes a fictitious point. Hence, the expression for the fictitious points are give by

$$
\begin{aligned}
& \bar{\zeta}_{i m}(E)=\zeta_{i m}(W)-2 \operatorname{LfU} U_{\text {re }}^{\prime}(C) / g \\
& \bar{\zeta}_{\mathrm{re}}(E)=\zeta_{\mathrm{re}}(W)-2 \operatorname{LfU} \mathrm{im}_{\text {im }}(\mathrm{C}) / g
\end{aligned}
$$

Then, the set of difference equations for the east boundary point, by using Eqs. (19a) and (19b) can be written as follows 
M. M. SIDJABAT

real part

$$
\begin{aligned}
& \frac{G(C)}{\omega L^{2}}\left(2 \zeta_{r e}(W)+\zeta_{r e}(N)+\zeta_{r e}(S)-4 \zeta_{r e}(C)\right)+ \\
& \frac{G y}{2 \omega L}\left(\zeta_{r e}(N)-\zeta_{r e}(S)\right)+\frac{F(C)}{\omega L^{2}}\left(2 \zeta_{i m}(W)+\zeta_{i m}(N)+\zeta_{i m}(S)-\right. \\
& \left.4 \zeta_{i m}(C)\right)-\frac{F y}{2 \omega L}\left(\zeta_{i m}(N)-\zeta_{i m}(S)\right)-\zeta_{i m}(C)= \\
& Q_{r e}(C)+T_{r e}(C)\left(\frac{G(C)}{\omega L^{2}}+\frac{G y(C)}{2 \omega L}\right)-T_{i m}(C)\left(\frac{F(C)}{\omega L^{2}}+\frac{F_{x}(C)}{2 \omega L}\right)(20)
\end{aligned}
$$

imaginary part

$$
\begin{aligned}
& \frac{G(C)}{\omega L^{2}}\left(2 \zeta_{i m}(W)+\zeta_{i m}(N)+\zeta_{i m}(S)-4 \zeta_{i m}(C)\right)+ \\
& \frac{G y(C)}{2 \omega L}\left(\zeta_{i m}(N)-\zeta_{i m}(S)\right)+\frac{F(C)}{\omega L^{2}}\left(2 \zeta_{r e}(W)+r_{r e}(N)+r_{r e}(S)-\right. \\
& 4 \zeta_{r e}(C)-\frac{F_{y}(C)}{2 \omega L}\left(\zeta_{r e}(N)-\zeta_{r e}(S)\right)+\zeta_{r e}(C)= \\
& Q_{i m}(C)+T_{r e}(C)\left(\frac{F(C)}{\omega L^{2}}+\frac{F_{y}(C)}{2{ }^{2}}\right)-T_{i m}(C)\left(\frac{G(C)}{\omega L^{2}}+\frac{C_{y}(C)}{2 \omega I}\right)(21)
\end{aligned}
$$

where $\mathrm{T}_{\text {re }}$ and $\mathrm{T}_{\mathrm{im}}$ are given by

$$
\begin{aligned}
& { }_{r e}^{T_{r e}}(c)=2 L f U_{r e}^{\prime}(c) / g \\
& T_{i m}(c)=2 L f U_{i m}^{\prime}(C) / g
\end{aligned}
$$

By the same procedure, the finite difference equations for the West, North and South boundaries can be obtained. 
Thus, in obtaining solutions for the surface displacement, $\zeta$,. in a region under consideration, Eqs. ( 17 ) and ( 18 ) must hold at every interior point, and Eqs. ( $15 \mathrm{a}, \mathrm{b})$ and ( $16 \mathrm{a}, \mathrm{b})$ must be satisfied at any appropriate fixed boundary point.

The matrix appearing in connection with the finite difference equations of a simple net-system, has a simple structure. It is a symmetric matrix and has many zero elements. This enables one to find the solution of the matrix by a triangularization method ( FORSVTHE \& WASOW 1960). However, if the real basin is taken into consideration, the irregularities of the boundaries and the bottom structure upset the symmetry, making the application of the triangularization method complicated. The other alternative is to employ a simple Gauss elimination method. However, elimination is ordinarily coded by actually storing the coefficient matrix for which the storage capacity of the computer becomes a problem. To overcome the storage problem, the scheme of inverting a matrix representing the equations under consideration has to be modified. A brief illustration is described as follows. Let A be a $\mathrm{n} \times \mathrm{n}$ matrix with coefficients represented by

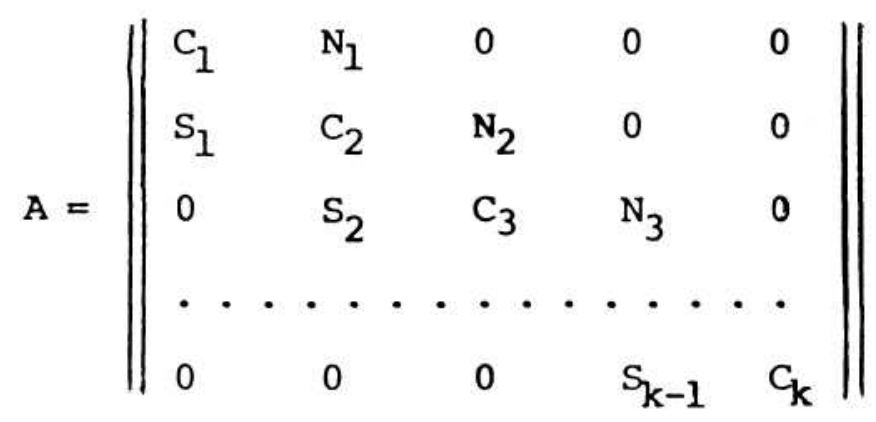

where each $C_{k}$ is an m-by-m matrix of sept-diagonal form (the non-zero elements are all on its main diagonal and the 3 adjacent diagonals on each side), and $\mathrm{S}_{\mathrm{k}}, \mathrm{N}_{\mathrm{k}}$ are tri-diagonal matrices (VARGA, 1962). This matrix resembles the matrix associated with the problem considered in this paper. The matrix $A$ then is reorganized and reduced into $A^{\prime}$, in such a way that the diagonal elements of $A$ are on the central column of $\mathrm{A}^{\prime}$ 


\section{M. SIDJABAT}

$$
A^{\prime}=\left\|\begin{array}{ccc}
0 & c_{1} & N_{1} \\
S_{1} & c_{2} & N_{2} \\
S_{2} & c_{3} & N_{3} \\
\cdot \cdot \cdot \cdot \cdot \cdot \cdot \\
S_{k-1} & c_{k} & 0
\end{array}\right\|
$$

By doing this, one is able to reduce the storage requirements in computer for inverting the matrix. The geometrical structure of the area under study and the grid size of the net system representing it are the main factors in determining the degree of reduction in the size of the matrix. When the matrix has been reduced, a new algorithm is used to invert the new matrix $\mathrm{A}^{\prime}$.

Although elimination methods are rarely used for solving elliptic difference equations, the author has examined a modified Gauss elimination which can be employed to solve the problem considered in this paper. An iterative scheme, such as an over-relaxation method (FOX 1950. YOUNG 1962) was also tried to solve the simultaneous equations. However, it was observed that computations converge very slowly (70 iterations) and still indicate significant discrepancies from the true solution (for the case of the rectangular basin) compared to the Gauss method.

From the computed $\zeta_{\text {re, }}$ and $\zeta_{\text {im }}$, the velocity can be obtained for each grid point. The real and imaginary parts of the velocity, $\mathrm{U}$ and $\mathrm{V}$, are given by

$$
\begin{aligned}
U_{r e}^{(i+l)}(C)= & -\frac{G^{i}(C)}{2 L}\left(\zeta_{r e}^{i}(E)-\zeta_{r e}^{i}(I)\right)+\frac{F^{i}(C)}{2 L}\left(\zeta_{i m}^{i}(E)-\right. \\
& \zeta_{i m}^{i}((N))-\frac{f}{g}\left(v_{r e}^{i}(C) G^{i}(C)-v_{i m}^{i}(C) F^{i}(C)\right) \\
U_{i m}^{(i+1)}(C)= & -\frac{G^{i}(C)}{2 L}\left(\zeta_{i m}(C)-\zeta_{i m}(W)\right)-\frac{F^{i}(C)}{2 L}\left(\zeta_{r e}^{i}(E)-\right. \\
& \left.\zeta_{r e}^{i}(I V)\right)-\frac{f}{g}\left(v_{i m}^{i}(C) G^{i}(C)+v_{r e}^{i}(C) F^{i}(C)\right)
\end{aligned}
$$




$$
\begin{aligned}
V_{r e}^{(i+1)}(C)= & -\frac{G^{i}(C)}{2 L}\left(\zeta_{r e}^{i}(N)-\zeta_{r e}^{i}(S)\right)+\frac{F^{i}(C)}{2 L}\left(\zeta_{i m}(N)-\right. \\
& \left.\zeta_{i m}(S)\right)+\frac{f}{g}\left(U_{r e}^{i}(C) G^{i}(C)-U_{i m}^{i}(C) F^{i}(C)\right) \\
U_{i m}^{(i+1)}(C)= & -\frac{G^{i}(C)}{2 L}\left(\zeta_{i m}(N)-\zeta_{i m}^{i}(S)\right)+\frac{F^{i}(C)}{2 L}\left(\zeta_{r e}(N)-\right. \\
& \left.\zeta_{r e}(S)\right)+\frac{f}{g}\left(U_{i m}^{i}(C) G^{i}(C)+U_{r e}^{i}(C) F^{i}(C)\right)
\end{aligned}
$$

At each stage of the calculation for each component, the computed velocity components are stored on disk or tape. When the computations for all components have been completed, these velocity components can be retrieved to evaluate the parameter $\lambda(\mathrm{x})$ which is then used for the next iteration calculations. The computations are repeated to obtain the solution for $\zeta_{n}$ and $U_{n}$ for each constituent, until the solutions converge to within a prescribed tolerance.

\section{COMPUTATIONS}

The numerical method described in the previous chapter is written in the Fortran IV language for IBM 360/365. Grid points representing the area under consideration are coded in such a way that the program can be applied to any form of basin. In order to verify this program, it is, first tested for a rectangular basin of constant depth. Using a linearized form of the friction term, the numerically computed solution for this rectangular basin can be compared to the analytical solution. The convergence of the computation is also investigated. Following this, computations for the simple model of the Bight of Abaco are performed in order to gain insight into the effect of the depth distribution and coastline configuration to the tidal motion; and also for further investigation of the convergence of the computation. Finally, computations with the real boundary conditions along the opening are conducted for the Bight of Abaco.

\section{COMPUTATIONS FOR A RECTANGULAR BASIN}

\section{Analytical Solution}

The analytical solution, which serves as the standard for comparison with the numerical method, is for the linear form of the hydrodynamical 


\section{M. SIDJABAT}

equations. For the sake of simplicity, the Coriolis force is not considered. The solution is developed for a one dimensional model, e.g., a channel with closed end and of uniform depth. The governing equations which arise from the linearization of ( $\mathrm{la}$ ) and ( $\mathrm{lb}$ ) for one dimensional problems are

$$
\begin{aligned}
& \frac{\partial \vec{u}(\vec{x}, t)}{\partial t}+\vec{u}(x, t)+g \frac{\partial \zeta(\vec{x}, t)}{\partial x}=0 \\
& \frac{\partial \zeta(\vec{x}, t)}{\partial t}+h \frac{\partial \vec{u}(\vec{x}, t)}{\partial t}=0
\end{aligned}
$$

where the boundary conditions are taken to be

$$
\begin{array}{ll}
\frac{\partial \zeta(0, t)}{\partial x}=0 & \text { for } x=0 \text { (closed end) } \\
\zeta(L, t)=\zeta_{L} e^{i \omega t} & \text { for } x=L \text { (open end) }
\end{array}
$$

The solution $\zeta(\vec{x}, \mathrm{t})$ can then be written as

$\zeta(\vec{x}, t)=\alpha_{A}\left(e^{\gamma_{r} e^{\vec{x}}} \cos \left(\omega t+\gamma_{i m} \vec{x}\right)+e^{-\gamma_{r} \vec{x}} \cos \left(\omega t-\gamma_{i m} \vec{x}\right)\right)$

where

$$
\begin{gathered}
\gamma_{\text {re }}=\frac{\omega}{\sqrt{\text { gin }}} \sqrt{\frac{\left(1+\frac{R}{\omega^{2}}\right)^{\frac{1}{2}}+1}{2}} \\
\gamma_{\text {im }}=\frac{\omega}{\sqrt{g h}} \sqrt{\frac{\left(1+\frac{R}{\omega^{2}}\right)^{\frac{1}{2}}-1}{2}}
\end{gathered}
$$

and

$$
A=\frac{\zeta_{L}}{\cosh \gamma_{r e} e^{\cos \gamma_{i m}{ }^{L}}}
$$


Numerical Solution

In order to verify the numerical method to be employed to compute tides in the Bight of Abaco and to test the iterative scheme described in the previous chapter, a rectangular basin of uniform depth was chosen as a model. The basin was open at one end and closed at the other. The size and depth of the model were taken as comparable with the real basin under study. The size of the basin was $46 \mathrm{x} 46 \mathrm{~km}$. The tidal constituent $\mathrm{M}_{2}$ was used as a forcing function along the opening. The numerical parameters used in computation are as follows:

$$
\begin{aligned}
\mathrm{L} & =5700 \mathrm{~m} . \\
\mathrm{h} & =5 \mathrm{~m} . \\
\mathrm{g} & =9.81{\mathrm{~m} \cdot \mathrm{sec}^{-2}} \\
\mathrm{r} & =0.003 \\
\mathrm{f} & =0.000064 \mathrm{rad} \mathrm{sec}^{-1}
\end{aligned}
$$

The rectangular basin is represented by 70 grid points. Ordinarily, the size of the matrix representing the equations considered here will be $140 \times 140$, however, by the suggested scheme, this size can be reduced to $140 \times 40$.

The amplitude at each grid point along the entrance was set equal to $17 \mathrm{~cm}$, which is taken as the average amplitude of $\mathrm{M}_{2}$ component along the entrance of the Bight of Abaco, and the phase is set equal to zero. By setting the Coriolis parameter $\mathrm{f}$ equal to zero, and allowing for the linear form of the frictional term, [The value of R (in 22a) used in this computation is an arbitrary value $(0.0003)$ which is irrelevant to the model] the result of the computations can be compared with the analytical solution which was obtained by the method discussed previously. The method of numerical computation was confirmed because at every grid point the computed tidal amplitude corresponds to the analytical solution. Between the corresponding points, the deviation in amplitude is less than $0.01 \mathrm{~cm}$ and in phase is less than 0.05 degree.

The computation with nonlinear friction was performed; it took 4 iterations to achieve the convergence of the solutions (after 4 iterations the difference between the solution of two consecutive computations is very small $(<0.1 \%)$ ), however, there is no way to justify whether the solution is the true one. The numerically computed solution for both computations with linear and non-linear friction were plotted in Fig. 2. In this figure, the computation with non-linear friction was first conducted. Then, from the resulting velocities, the average along the channel was used to evaluate the coefficient of friction for the computation with linear 


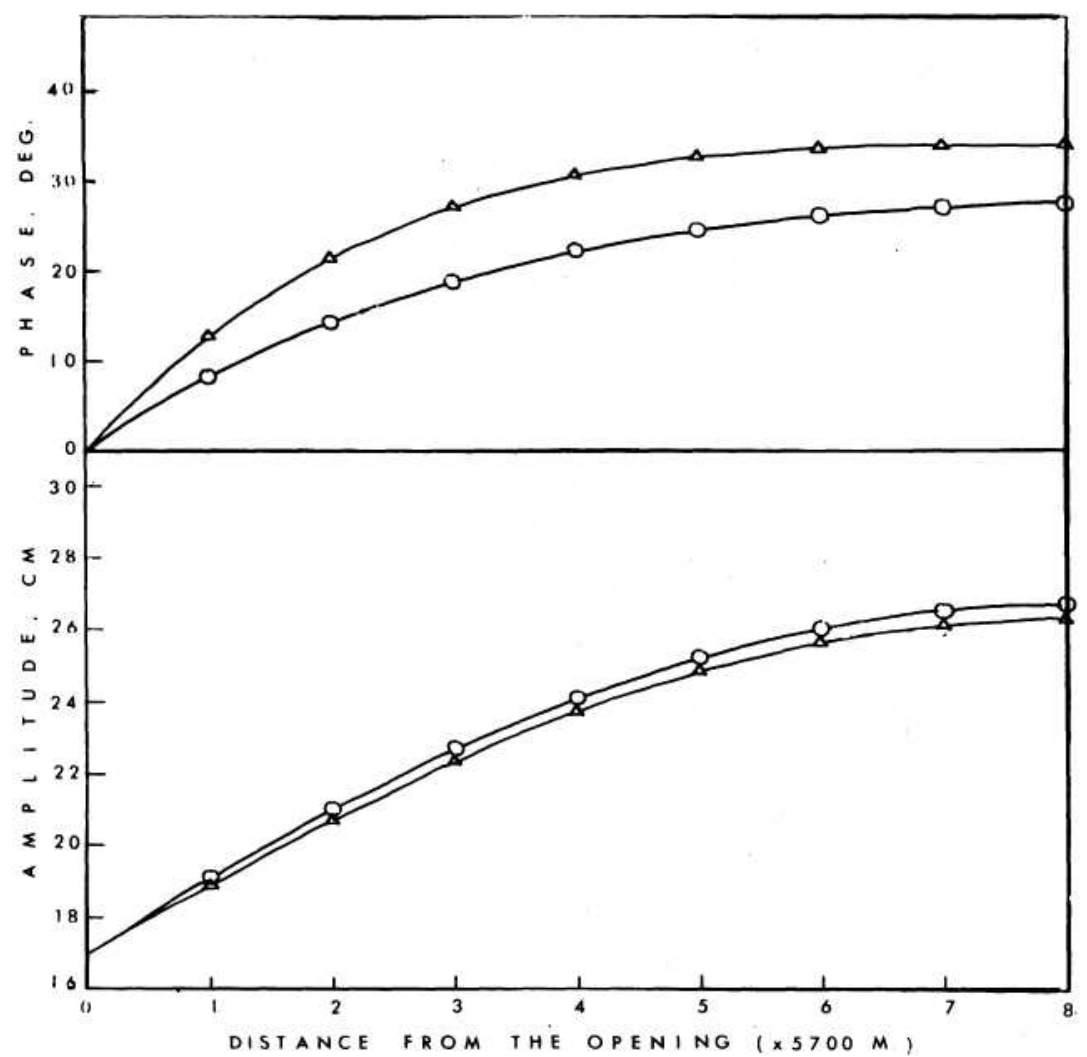

Figure 2. Phase and amplitude distribution of the computed solutions for M2 corresponding to a rectangular basin with uniform depth. The lines with triangles are for the computation with linear friction and the lines with the circles are for the nonlinear friction.

friction. As one may observe, there is a slight difference between the amplitude distribution of the two computations, but a more significant difference is observed in the phase distributions. If the depth of the basin is set larger than $5 \mathrm{~m}$, this difference becomes less significant. Computations with and without the Coriolis force were also made; the results indicate that the effect of this force is very small (1 to $3 \%)$ compared to the total tidal amplitude.

\section{COMPUTATIONS FOR THE BIGHT OF ABACO}

\section{Simplified Model}

The grid system for the Bight of Abaco can be seen from Fig. 3. In setting this grid system, several assumptions were made. The Bight 


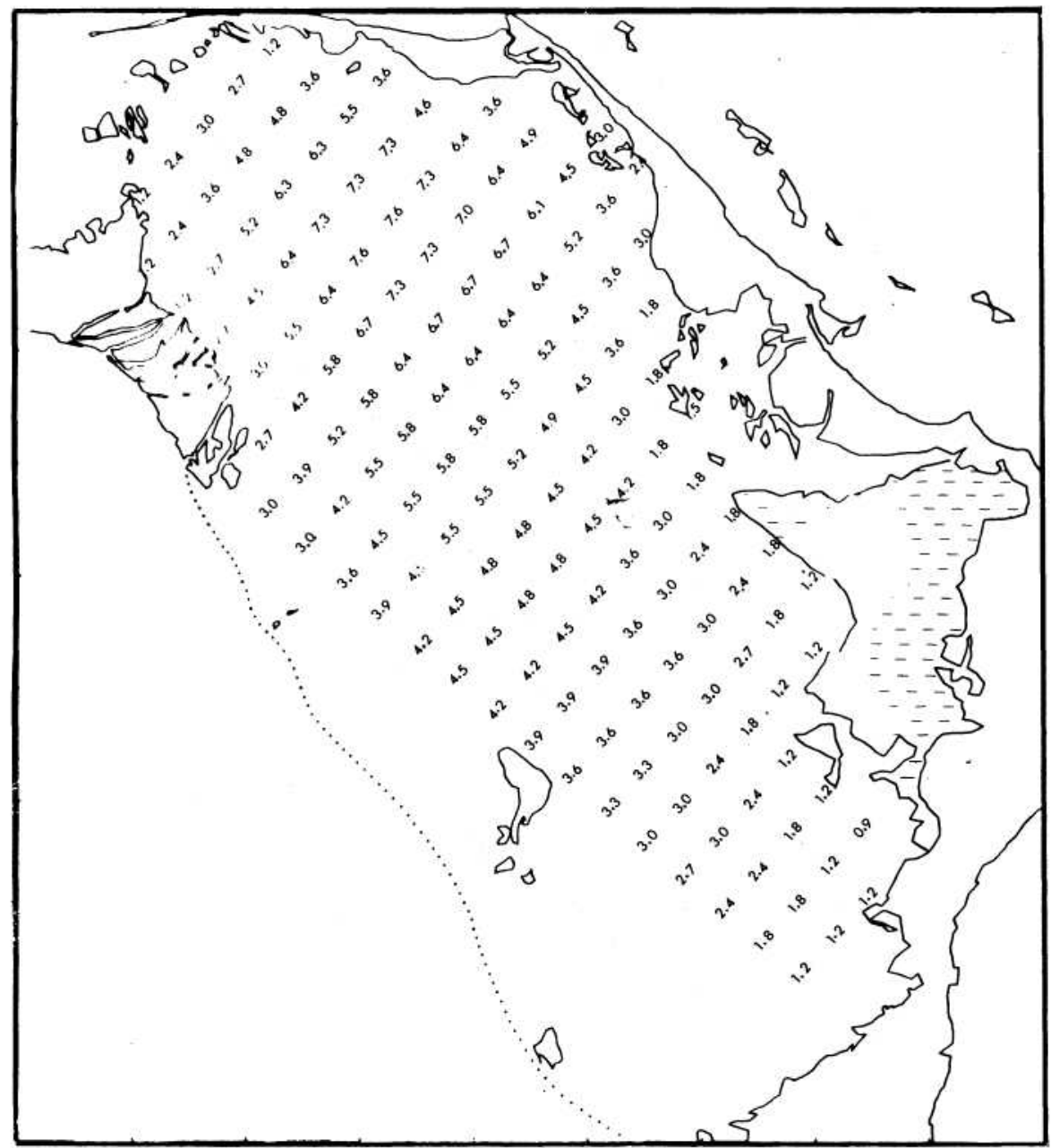

Figure 3. Depth distribution of the Bight of Abaco.

was assumed to be closed at the northern end, since the channels between the islands there are narrow and shallow. In other words, the inflow and outflow of water between these islands due to the tides was assumed negligible. This assumption is supported by the tide observation at station 20 where the amplitudes and phases differ considerably from those observed at the northern end of the Bight. For practicality, this opening should also be treated as closed, for the size of it is less than the mesh size' of the grid system $(4572 \mathrm{~m})$ used in the numerical modeling of the region. 


\section{M. SIDJABAT}

The above assumption was also adopted for the southeast boundary of the Bight, where the marls are located. Then, the area under consideration has only one opening, that is the western entrance. A boundary line was drawn along this entrance, in such a way that all the complicated land distribution along the entrance lay outside of the net system. The numerical modeling of the area would be too complicated if this line had to be drawn outside of the land areas, although the setting of the boundary values might be easier. The numerical treatment of this type of problem has apparently not been investigated.

The net-system has 155 grid points, 16 of which are located along the free boundary. The numerical values used here are practically the same as in the previous model, except for the mesh size. The boundary values for the grid points along the boundary are made uniform; the amplitude is $17 \mathrm{~cm}$, and the phase is set equal to zero. The depth is taken to be uniform.

For this model, it is observed that the number of iterations required for convergence of the solutions is larger than that for the rectangular basin. This may be mainly due to the irregularity of the boundaries. In order to speed the convergence of the solution, a new algorithm was introduced in approximating the non-linear form of the frictional term. In place of Eq. (3.7), the equation

$$
\begin{aligned}
& i \omega \vec{U}_{n}(i+1)+\frac{r}{h}\left(p_{1} \lambda^{i}(\vec{x})+p_{2} \lambda^{i-1}(\vec{x})\right) \vec{U}_{n}(i+1)+ \\
& 2 \Omega \times U_{n}^{i}+g \vec{\nabla} \zeta_{n}(i+1)=0
\end{aligned}
$$

was used. The parameters $\mathrm{p}_{1}$ and $\mathrm{p}_{2}$ are known as weight parameters, which satisfy the relation

$$
p_{1}+p_{2}=1
$$

The effect of using the best combination of $\mathrm{p}_{1}$ and $\mathrm{p}_{2}$ reduces the number of iterations necessary for convergence of the solution by a factor 4. Fig. 4 illustrates the number of iterations for 3 different combinations of $\mathrm{p}_{1}$ and $\mathrm{p}_{2}$. It can be seen that $\mathrm{p}_{1}=0.75$ and $\mathrm{p}_{2}=0.25$ is the best combination of the three. There values were used for the rest of the computations.

A computation was carried out using the frictional coefficient $r$ equal to 0.003 . From the computed tidal amplitude and phase, the cophase and corange lines were constructed for the model. It was found that the tidal amplitude increases as the tide approaches the northern 
MODELLING OF TIDES IN BASIN

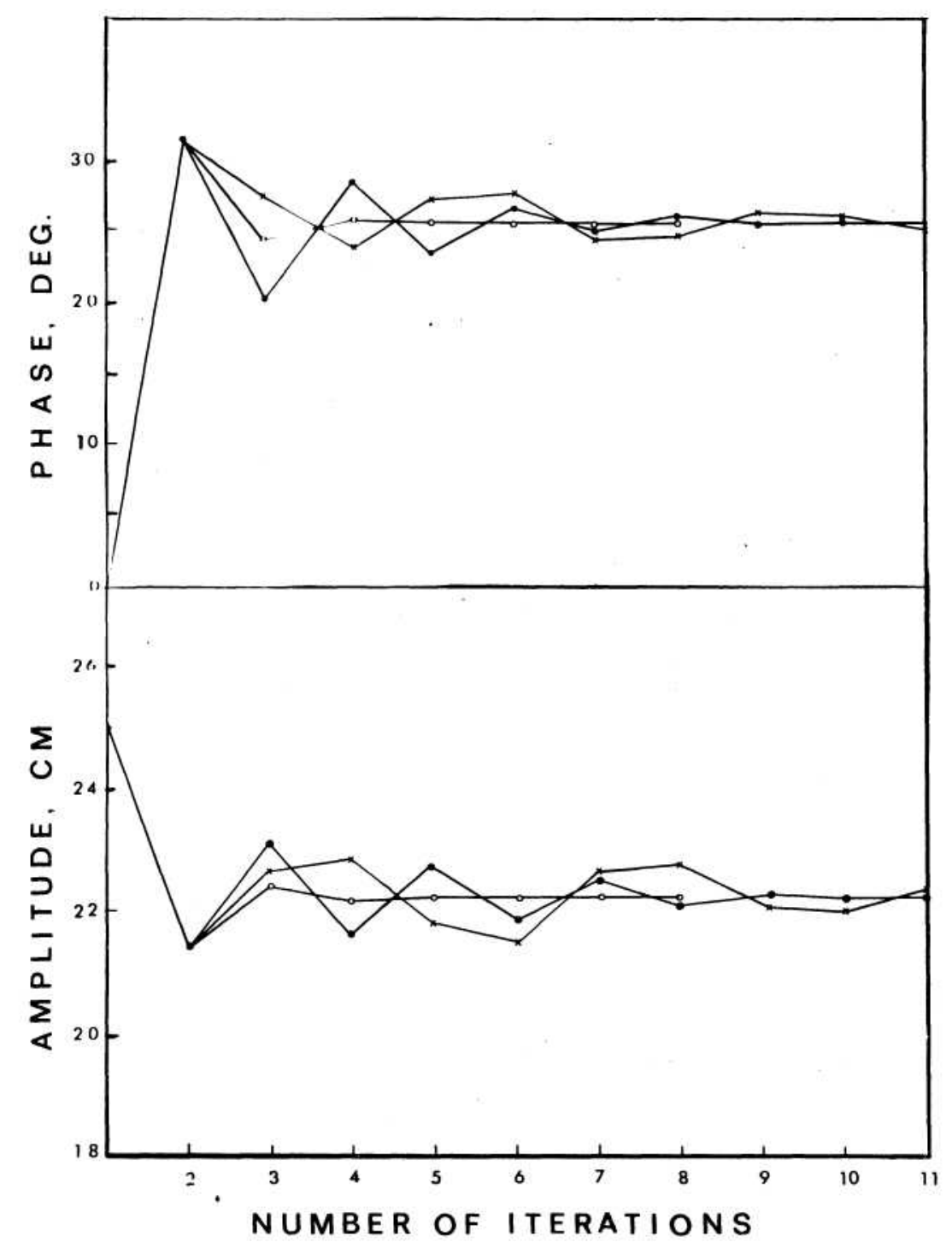

Figure 4. Rate of convergence of the solution for the amplitude and phase for a model basin at a particular grid point (at the middle of the basin) for different combinations of pi and $\mathrm{p}_{2}$ :

- $\mathrm{p}_{1}=1.0, \mathrm{p}_{2}=0.0$
$x: \mathrm{p}_{1}=2 / 3, \mathrm{p}_{2}=1 / 3$
$\varnothing: \mathrm{p}_{1}=3 / 4, \mathrm{p}_{2}=1 / 4$ 


\section{M.M. SIDJABAT}

boundary of the model, and at the same time the rate of change in phase decreases. This phenomenon is mainly a result of reflection of the tidal wave by the corresponding boundary. Near the boundary, the size of the amplitude is twice as large as the size of the incoming wave. As the reflected wave propagates away from the reflecting boundary, the amplitude decreases again due to the damping effect of the bottom on the model. If a frictionless model is considered, a pure standing wave is obtained with zero phase everywhere in the model.

A computation was also performed for the model where the real depth was considered. The boundary conditions are the same as before. The use of real depth in the computation results in significant changes in the amplitude and phase distributions. One can see that, due to the small depth along the entrance, the tidal amplitude drops drastically, but as the tide enters the deeper part of the model its amplitude increases. In this model the smallest amplitude is observed right after the entrance. This can be explained by similar arguments to those considered for the model of uniform depth, but in this case the reflected waves apparently have small effects at the regions near this entrance. The difference in phase distribution in the 2 models is also noticeable. Due to the shallower depth near the coast for the model with real depth, the phases are retarded compared to the model with uniform depth. Both models demonstrate that the coast and bottom configuration have very significant effects on the propagations of tides.

\section{Real Model}

The grid system used for this model is essentially the same as the one for the simple model. The real depths are taken into consideration; the depth distribution which was obtained from the Hydrographic Chart (H.O. 5990) -published by U.S. Naval Oceanographic Office in 19H3, is illustrated in Fig. 3. Again, the numerical parameters used here are also identical to those in the previous model except for the number of tidal components considered in the computations. The boundary values along the opening were obtained from the observed tides from the 5 tidal stations that were installed along this entrance. The proper setting of the tidal constants along this opening is the most important factor in obtaining an accurate solution. These 5 stations were not exactly located along the line that is used as a free boundary of the model. Since the bottom configuration along this entrance is so complicated and because of the limited number of observations in the neighborhood of the opening, it would be difficult to obtain the boundary values based on these observations alone. 
The following procedure was therefore adopted to carry out the determination of the boundary values for the numerical computations. First, corresponding to each of these 5 stations, a standard point was determined along the free boundaries of the model in such a way that the point was the projection of the station to the opening line of the grid system. Then, the approximated tidal constants at these 5 points were determined by visual inspection, from which the boundary values for each grid point along the opening can be obtained by cubic spline interpolation (through the aid of curves). This interpolation was performed for each tidal component both in amplitude and phase. Using these initial guesses of the boundary values, the computations were performed as prescribed previously. These computations give the solution of each component for all grid points in the model. From these results., the computed tidal amplitudes and phases for each boundary station were obtained by polynomial interpolation. By doing this, the numerically computed tidal constants can be compared to the observed ones. It is recognized that for this stage of calculation, the difference between the computed and observed tides are significant. The source of these large deviations is due mainly to the improper setting of the boundary condition along the opening. These boundary conditions can be improved by readjusting the tidal constants at those 5 standard points in accordance with the numerical results. Following this, the computations were performed again, and it was observed that the results of computations were improved. The above procedure can be repeated several times until the observations and computations at the stations along the entrance agree within a prescribed tolerance. In the computations considered here, the third readjustment of the boundary value was found to be satisfactory. This final setting of the boundary conditions is shown in Fig. 5. The difference between the computed and observed values for both amplitude and phase for the stations along the opening is less than 19f. For the adjustment of the boundary condition the value of $r=0.003$ was used on the computations.

When the proper boundary condition along the opening had been determined, a number of computations using different values of $r$ (from 0.002 to 0.006 ) were conducted. It appears that this small) change of the value of $r$ does not require the readjustment of the boundary conditions.

It was observed that the best value for the frictional coefficient $r$ is 0.0034 . Using this value, the tidal amplitude and phase are computed for the 5 tidal components considered in this study. From the results, the corange and cophase for each constituent were constructed (Figs. 6 - 7). One can observe that the appearance of the amplitude and phase distribution depends upon the period of the tidal component. The semi- 
M. M. SIDJABAT

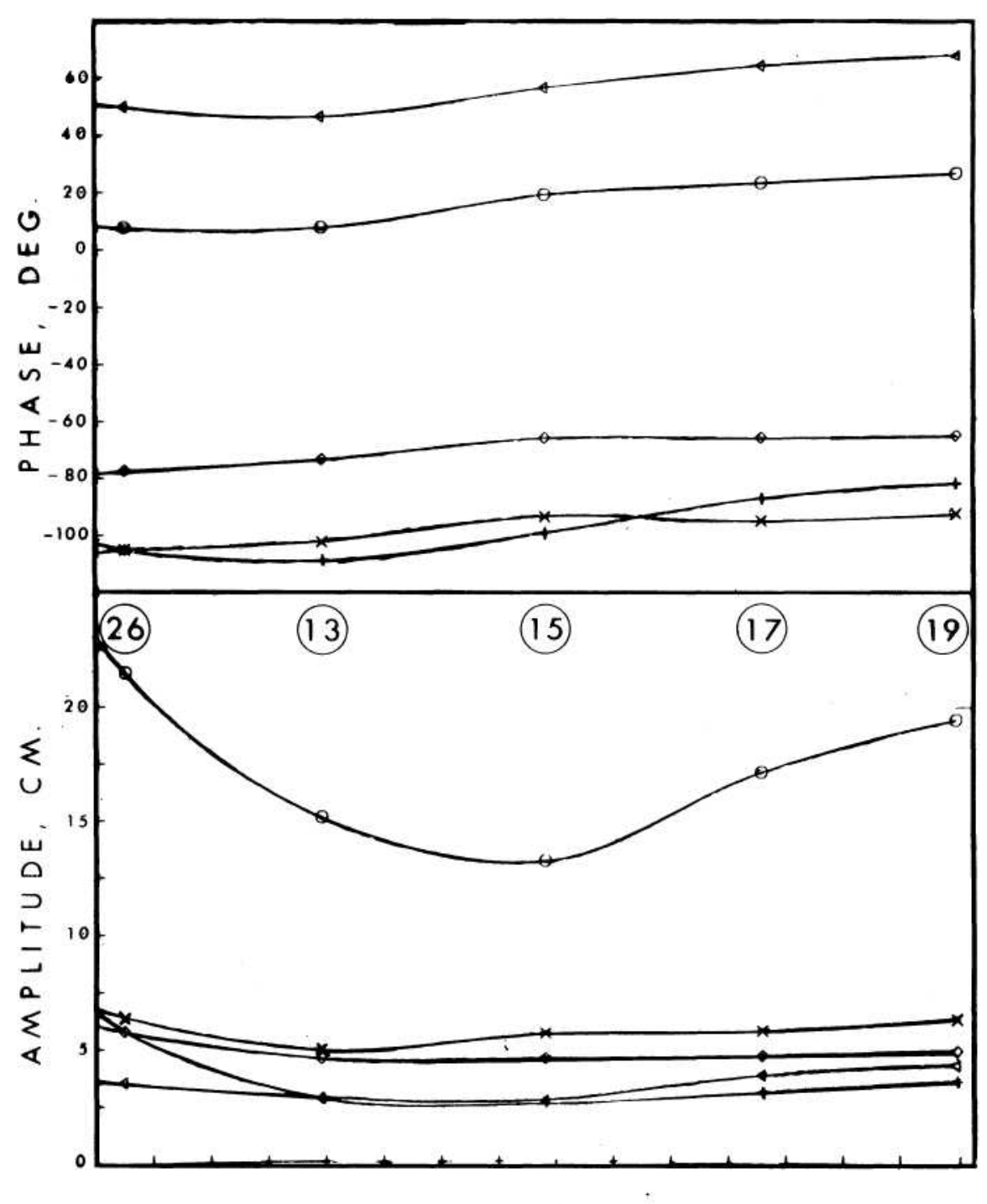

GRIO POINTS ALONG THE OPENING

Figure 5. The final setting of the amplitude and phase distribution of the 5 constituents along the opening based on the observed and computed tides at the stations that are located along the entrance. The symbols used in this figure are defined as follows:

$$
\begin{array}{ll}
\nabla-\mathrm{M}_{2} & \text { component } \\
\triangleleft-\mathrm{S}_{2} & \text { component } \\
\diamond-\mathrm{N}_{2} & \text { component } \\
\times-\mathrm{K}_{1} & \text { component } \\
+-\mathrm{O} 1 & \text { component }
\end{array}
$$




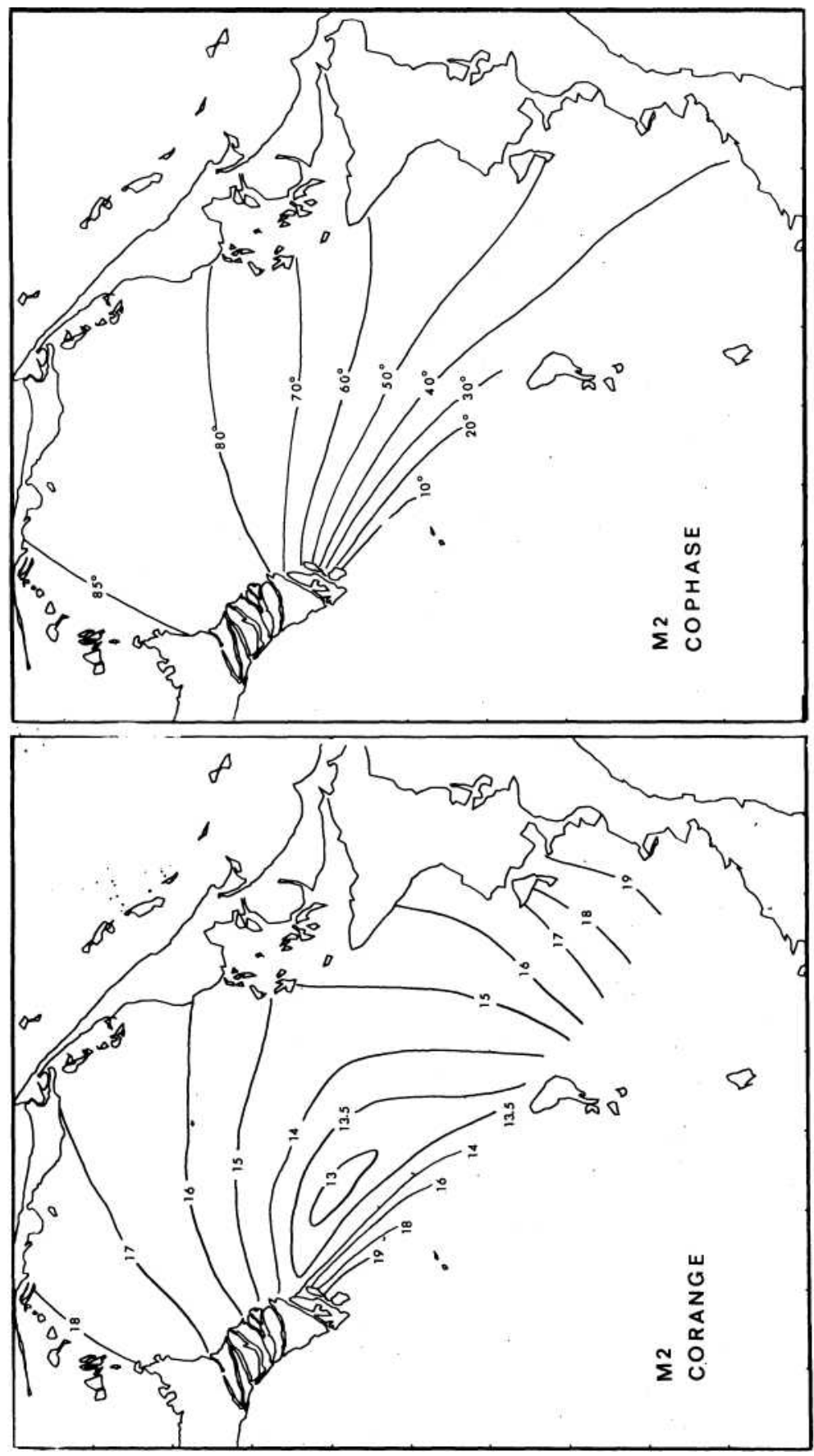

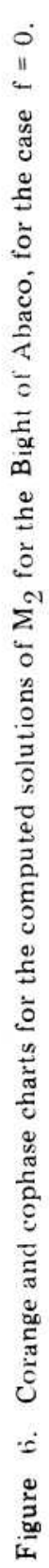




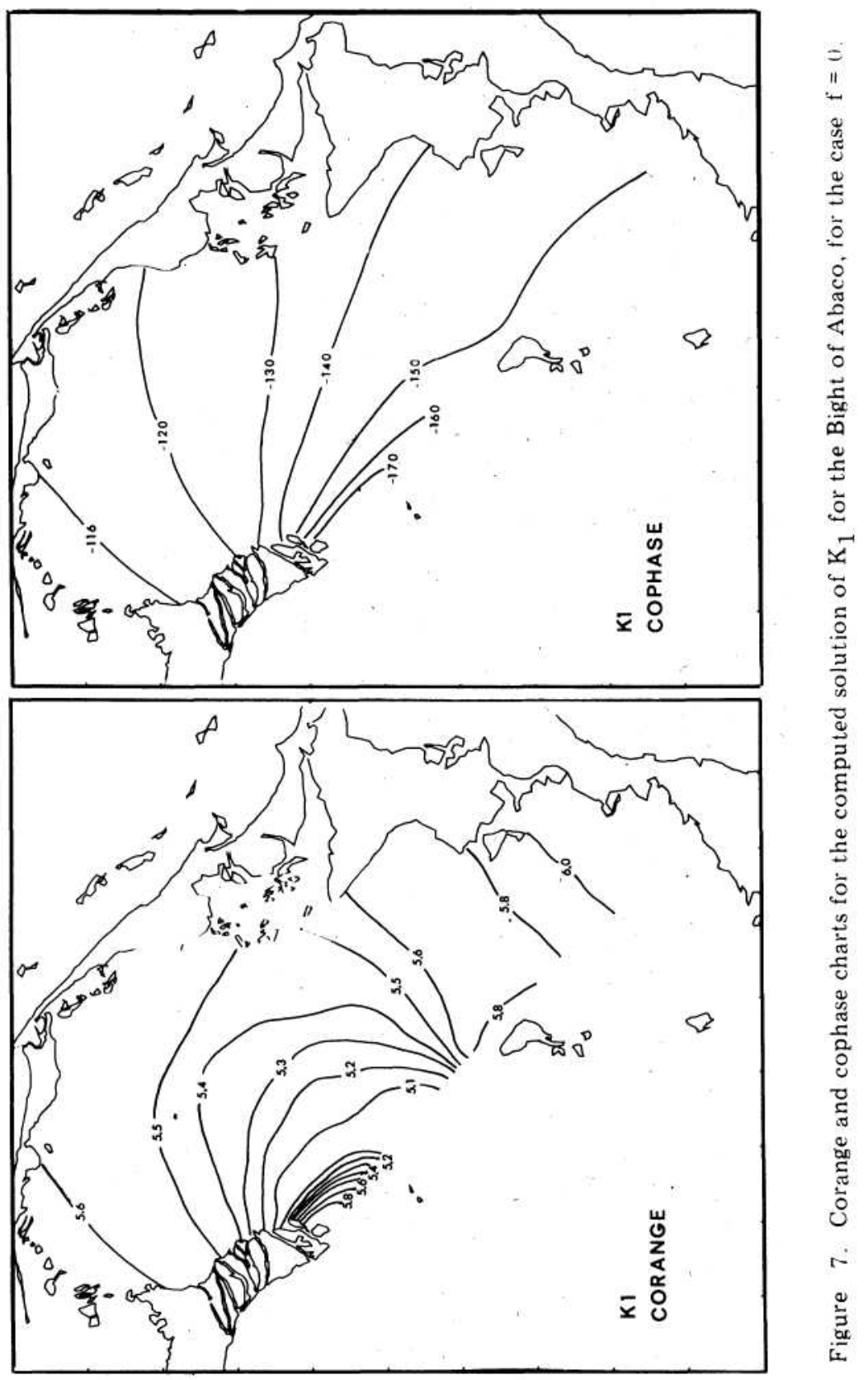


diurnal components have the same qualitative distribution and the same thing holds true for the diurnal components. However, there is a noticeable difference between corange of $\mathrm{K}_{1}$ and $\mathrm{O}_{1}$ components. This difference is mainly due to the different amplitude distribution of $\mathrm{K}_{1}$ and $\mathrm{O}_{1}$ along the opening (Fig. 5), which in turn may result from errors in the harmonic analysis for these constituents due to the shortness of the records and the meteorologically induced noise.

\section{DATA ANALYSIS}

\section{Tidal Observations}

The tidal observations described in this paper were made in the Bight of Abaco, Bahamas, by R. L. Snyder of Nova University and J. H. Filloux of Gulf General Atomic Corporation, San Diego. A chart of the Bight is shown in Fig. 8. Tide gauges designed and built by J. H. Filloux (1969) were used. These were shallow water versions of a small, high-resolution, self-contained and bottom mounted instrument designed to record oceanic tidal fluctuations anywhere in the ocean.

The observations were conducted from February 5 to March 24, J969; and 15 tidal gauges were installed throughout the region. A second series of observations was run from July 10 to August 17, 1969. For accurate positioning of the tidal gauges, Decca Hi-Fix was used. Five gauges were placed on the inside entrance of the Bight, the information from which was used to set the boundary condition along the opening for the numerical computation. Inside the Bight, 10 gauges were installed. The observed tidal amplitudes and phases obtained from these gauges were used as comparisons to the computed ones. In order to gain a qualitative understanding of the tidal propagation through the northern and western openings, 4 gauges were planted outside the corresponding entrances. In addition to the tide gauges, four weather stations were installed. The observations were made from R.V. GULFSTREAM and R.V. L.F.R. BEL'LOWS, research vessels of the Physical Oceanographic Laboratory of Nova University.

\section{Harmonic Analysis}

As mentioned previously, the surface elevations were inferred from the pressure changes measured at the bottom of the sea. Hence, the atmospheric pressure fluctuations were also incorporated in the tidal records, e.g., 


\section{M. SIDJABAT}

$$
\mathrm{P}=\operatorname{pg}(\mathrm{h}+\zeta)-\mathrm{p}_{\mathrm{a}}
$$

where $p$ is the pressure recorded by a tidal gauge and $p_{a}$ is the atmospheric pressure which was also recorded simultaneously at the surface of the sea. However, since the only significant period in the atmospheric fluctuations that is also studied in the tidal fluctuations is $S_{2}$ (period of 12

hours), the above correction has been applied directly to the analyzed constants for this constituent. It was assumed that the atmospheric pressure is uniform everywhere in the Bight of Abaco. Therefore, information from only one record of atmospheric pressure was needed.

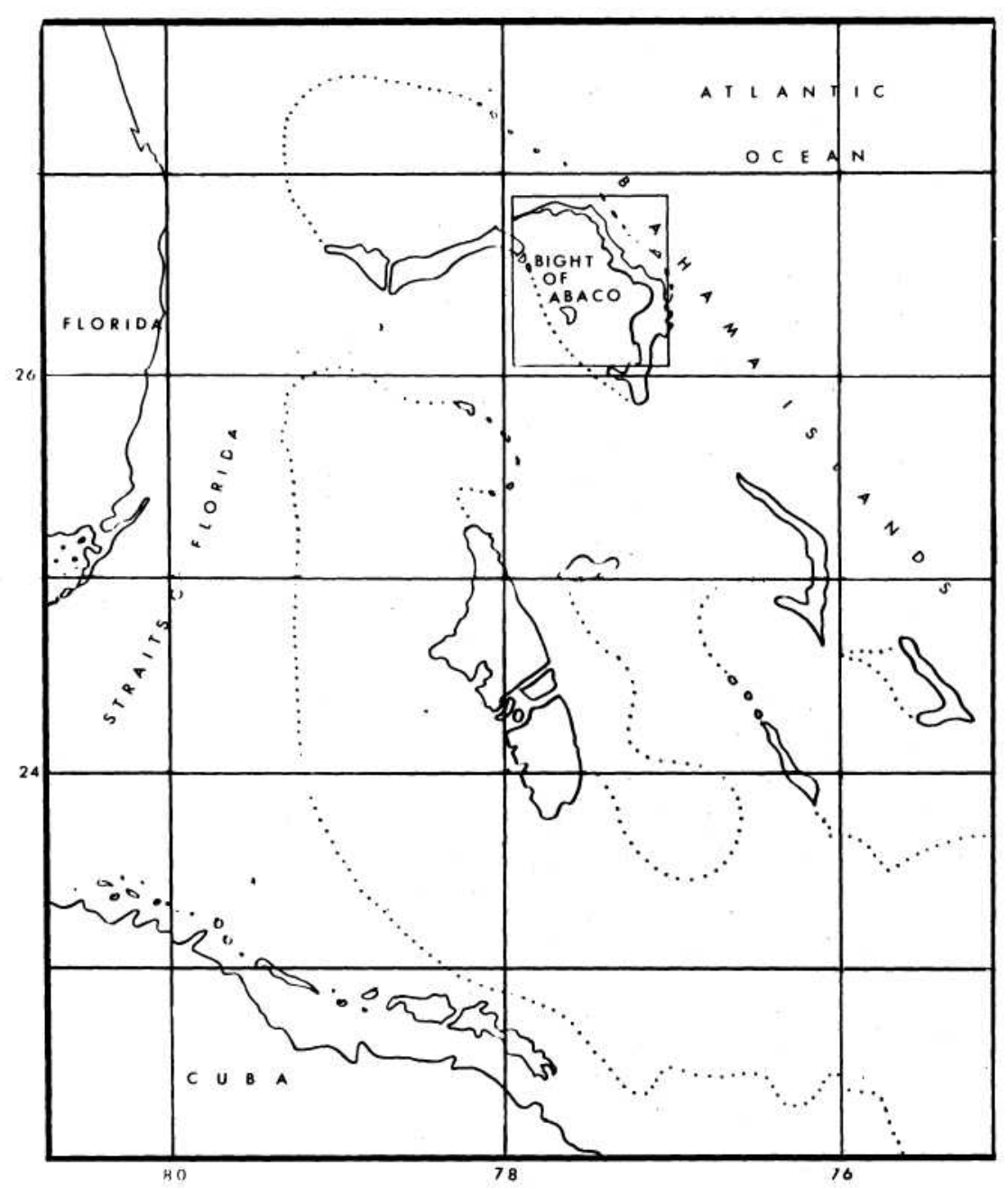




\section{MODELLING OF TIDES IN BASIN COMPARISON}

\section{BETWEEN NUMERICAL COMPUTATIONS AND OBSERVATIONS}

In order to evaluate the hydrodynamical model considered in this study, it is necessary to compare numerical computations with observations. There are several ways to perform this comparison, and one of these is to calculate the variance between the computed tides and the observed tides. This variance will be defined as the mean square value of the difference between the computed tides and the observed ones. This variance can be represented in four different ways.

1. Mean square difference due to a single component at individual station, e.g.,

$$
v_{n}(\vec{x})=\frac{1}{2}\left[\zeta_{n}^{c}(\vec{x})-\zeta_{n}^{O}(\vec{x})\right]^{2}
$$

where as before the operator [ ] denotes amplitude, $\zeta_{n}^{c}$ and $\zeta_{\mathrm{n}}^{\mathrm{O}}$ are the computed and observed tides respectively.

2. Mean square difference due to all components at individual station, e.g.,

$$
v(\vec{x})=\frac{1}{\Sigma} \sum\left[\zeta_{n}^{c}(\vec{x})-\zeta_{n}^{O}(\vec{x})\right]^{2}
$$

3. Average mean square difference for a single component over all stations, e.g.,

$$
v_{n}=\frac{1}{2 k} \sum_{k}\left[\zeta_{n}^{c}\left(\vec{x}_{k}\right)-\zeta_{n}^{o}\left(\vec{x}_{k}\right)\right]^{2}
$$

4. Average mean square difference due to all components over all stations for different value of r, e.g.,

$$
\mathrm{V}=\frac{1}{2 \mathrm{k}} \sum_{\mathrm{k} n} \sum_{n}\left[\zeta_{\mathrm{n}}^{\mathrm{c}}\left(\overrightarrow{\mathrm{x}}_{\mathrm{k}}\right)-\zeta_{\mathrm{n}}^{\mathrm{O}}\left(\overrightarrow{\mathrm{x}}_{\mathrm{k}}\right)\right]^{2}
$$

The value of the variances discussed above are given in Tables 1 to IV. The tabulated variances in Tables I and II indicate that the computed tides are in good agreement with the observed ones both in amplitude and phase for the two given components. It is observed that the variance varies from station to station as well as from component to component. A significant deviation is noted for station 8. Although the computed amplitudes seem to agree very well with the observed amplitudes, the 
M. M. SIDJABAT

Table I. Variance due to $\mathrm{M}_{2}$ for each station

\begin{tabular}{|c|c|c|c|r|r|}
\hline \multirow{3}{*}{ Stations } & \multicolumn{2}{|c|}{ Amplitude (cm) } & \multicolumn{2}{c|}{ Phase (deg.) } & Variance \\
\cline { 2 - 5 } & & & & & \\
& Observed & Computed & Observed & Computed & (cm2) \\
\hline 1 & 18.77 & 17.78 & 94.36 & 87.84 & 2.88 \\
2 & 18.06 & 17.36 & 92.39 & 84.65 & 2.84 \\
4 & 17.13 & 16.82 & 90.30 & 83.70 & 2.29 \\
5 & 16.36 & 16.55 & 87.21 & 81.57 & 1.14 \\
6 & 16.64 & 15.77 & 94.28 & 81.75 & 6.27 \\
7 & 16.00 & 15.38 & 89.79 & 79.01 & 4.31 \\
8 & 15.08 & 13.06 & 92.81 & 59.48 & 35.96 \\
9 & 12.67 & 13.20 & 78.55 & 63.50 & 5.85 \\
10 & 14.51 & 14.59 & 68.90 & 65.12 & 0.43 \\
13 & 16.90 & 16.94 & 2.15 & 2.85 & 0.02 \\
15 & 13.45 & 13.42 & 29.24 & 29.77 & 0.01 \\
17 & 17.14 & 17.23 & 33.40 & 33.51 & 0.01 \\
19 & 19.67 & 19.67 & 40.41 & 50.12 & 0.01 \\
26 & 27.92 & 27.96 & 8.45 & -9.27 & 0.06 \\
& & & & & \\
\hline
\end{tabular}

Table II. Variance due to $\mathrm{K}_{1}$ for each station

\begin{tabular}{|c|c|c|c|c|c|}
\hline \multirow[t]{2}{*}{ Stations } & \multicolumn{2}{|c|}{ Amplitude $(\mathrm{cm})$} & \multicolumn{2}{|c|}{ Phase (deg.) } & \multirow{2}{*}{$\begin{array}{r}\text { Variance } \\
\left(\mathrm{cm}^{2}\right)\end{array}$} \\
\hline & Observed & Computed & Observed & Computed & \\
\hline 1 & 5.19 & 5.63 & -125.36 & -116.86 & 0.42 \\
\hline 2 & 5.07 & 5.60 & -125.25 & -117.83 & 0.38 \\
\hline 4 & 5.16 & 5.55 & -126.73 & -118.58 & 0.37 \\
\hline 5 & 5.04 & 5.52 & -131.75 & -118.97 & 0.80 \\
\hline 6 & 5.09 & 5.45 & -125.00 & -119.50 & 0.19 \\
\hline 7 & 5.18 & 5.42 & -125.38 & -120.58 & 0.13 \\
\hline 8 & 5.66 & 5.22 & -108.85 & -130.05 & 2.10 \\
\hline 9 & 5.42 & 5.29 & -129.22 & -128.38 & 0.02 \\
\hline 10 & 5.95 & 5.45 & -133.74 & -126.50 & 0.39 \\
\hline 13 & 5.23 & 5.12 & -159.30 & -159.51 & 0.01 \\
\hline 15 & 5.73 & 5.80 & -140.22 & -139.90 & 0.01 \\
\hline 17 & 5.84 & 5.86 & -143.00 & -142.94 & 0.01 \\
\hline 19 & 6.44 & 6.43 & -138.09 & -138.23 & 0.01 \\
\hline 26 & 6.94 & 7.27 & -133.74 & -126.50 & 0.26 \\
\hline
\end{tabular}


MODELLING OF TIDES IN BASIN

Table III. Variance due to each component over all stations

\begin{tabular}{|c|c|}
\hline Component & $\begin{array}{c}\text { Average Mean Square Deviation } \\
\left(\mathrm{cm}^{2}\right)\end{array}$ \\
\hline $\mathrm{M}_{2}$ & 4.32 \\
$\mathrm{~S}_{2}$ & 0.42 \\
$\mathrm{~N}_{2}$ & 0.70 \\
$\mathrm{~K}_{2}$ & 0.35 \\
$\mathrm{O}_{2}$ & 0.11 \\
\hline
\end{tabular}

Table IV. Variance due to the 5 components for each station

\begin{tabular}{|c|c|}
\hline Station & Mean Square Deviation $\left(\mathrm{cm}^{2}\right)$ \\
\hline 1 & 5.05 \\
2 & 4.63 \\
4 & 4.92 \\
5 & 3.14 \\
6 & 6.58 \\
1 & 5.86 \\
8 & 41.61 \\
9 & 7.08 \\
10 & 2.71 \\
13 & 0.09 \\
15 & 0.05 \\
17 & 0.06 \\
19 & 0.06 \\
26 & 0.42 \\
\hline
\end{tabular}

differences in phase are very noticeable. These discrepancies are probably the failure of the numerical model to adequately represent the complex bottom topography just south of the Grand Bahama Island. On the other hand, observations and data analysis may contribute errors to a certain degree. From Table III, one can see that the relative magnitude of the variance for $\mathrm{S}_{2}$ and $\mathrm{N}_{2}$ are larger than for the other 3 components. This fact could be due to several factors other than numerical modeling. The amplitude of $\mathrm{S}_{2}$ and $\mathrm{N}_{2}$ are small compared to the amplitude of $\mathrm{M}_{2} . \mathrm{K}_{1}$ and $\mathrm{O}_{1}$ and they are also small compared to the noise level 
M. M. S

SIDJABAT

accompanying the observations. A small error in the observed values for these components may produce a large proportional deviation from the computed values. It is also relevant to point out that in determining the amplitude and phase of the tidal records, a conventional harmonic analysis was employed without making any further correction for the interference of other tidal constituents that have frequencies in the neighborhood of the principal components. 'These neighbor components were not taken into account in the harmonic analysis because of the noise level and record length. Although the analyzed constants for $\mathrm{K}_{1}$ really represent a combined curve for $K_{1}$ and $P_{1}$ (and similarly $S_{2}$ for $S_{2}$ and $\mathrm{K}_{2}$ ), this does not affect the principal aspect of this study if the observations are simultaneous for the whole region. However, the data for stations 8 and 26 are about three months later than the others and therefore the data for these stations are not as reliable in the calculations. Fortunately, station 26 is located at a relatively non-sensitive position of the area. Since the contribution of station 26 to the overall solution in the Bight is therefore reduced, this also suppresses the effect of the error on the solution. Also, $\mathrm{M}_{2}$, the principal constituent which mainly determines the frictional force, is not affected by the change in period. Error estimates for the observed tidal amplitudes and phases were not made because of time limitations.

In the following, several important factors that bear on the accuracy of the hydrodynamical and numerical modeling are discussed.

1. The hydrodynamical equations that are used in the model have been simplified to some extent in order to simplify the numerical modeling.

2. The assumption that

$$
\vec{n} \cdot \vec{u}=0
$$

along the hypothetical boundaries does not correspond to the actual conditions.

3. The inaccuracy of the depth distribution.

4. Since the numerical modeling entails the representation of a continuous system by a discrete one, an error can be introduced. This error can be particularly significant if the scale of the change in the variables of the problem is locally small compared to the mesh size.

5. The computations are subject to round-off error. Since the iterative scheme is used, each computed value will influence the values which are yet to be determined. In reality, this distribution of errors is often more or less a random process, and the effect of the errors will partially cancel out. 
In order to determine the optimum value for the friction coefficient $r$, the variance $\mathrm{V}$ was calculated for value of $\mathrm{r}$ in the range 0.002 to 0.006 . Figure 9 illustrates the distribution of this variance with respect to the numerical value of $r$. It is noted that, the minimum variance is given by a frictional coefficient r. 0.0034. As one may observe, the distribution of the variance is not symmetrical with respect to the optimum value of $r(=0.0034)$. A similar situation was observed by ISOZAKI and UNOKI (1964) in their Tsunami studies in Tokyo Bay.

Computations were also performed with different values of $r$ without including the Coriolis force. The resulting variance versus $\mathrm{r}$ can also be seen in Fig. 9. Around the point of minimum variance, the inclusion of the Coriolis force corresponds to $\pm 10 \%$ variation of resistance coefficient $r$. Since the effect of phase deviation is minimal at this point, this result is in reasonable agreement with the dimensional analysis given earlier.

\section{SUMMARY AND CONCLUSION}

The present study has demonstrated that the numerical modeling of tides in a shallow basin, where the friction term is non-linear, can be performed by a modified elliptic method. This method can readily produce the solution for each tidal component and at the same time preserve the nature of the non-linearity of the system and the interaction among the tidal components. In applying the iterative scheme in approximating the non-linear term, the convergence and stability of the computation do not appear to be a real problem. Other non-linear terms in the equations could be included in the computation without major difficulty; however, as has been demonstrated in this study these terms are small compared to the leading terms in the equations.

The viability of the method considered in this paper is indicated by the fact that the results of computations using a coefficient of friction $\mathrm{r}=0.0034$ give good agreement with observations for all components and over all stations. This frictional coefficient is very close to the value $(0.003)$ that is commonly reported by many workers.

Computations,, both for the simple model and for the real basin, indicate ${ }^{4}$ that the bottom topography as well as the coastline configuration have a very significant effect on the tidal propagation. Thus, it is necessary to represent the geometry of the area under consideration as accurately as possible, which requires (I) accurate information of the depth distribution of the area and (2) a small grid size for the net system that models the area. However, if a version of the elimination method is to be employed in inverting the matrix associated with the problem, then the storage capacity of the computer becomes a limiting factor. A better technique of inverting this matrix may overcome this problem. 


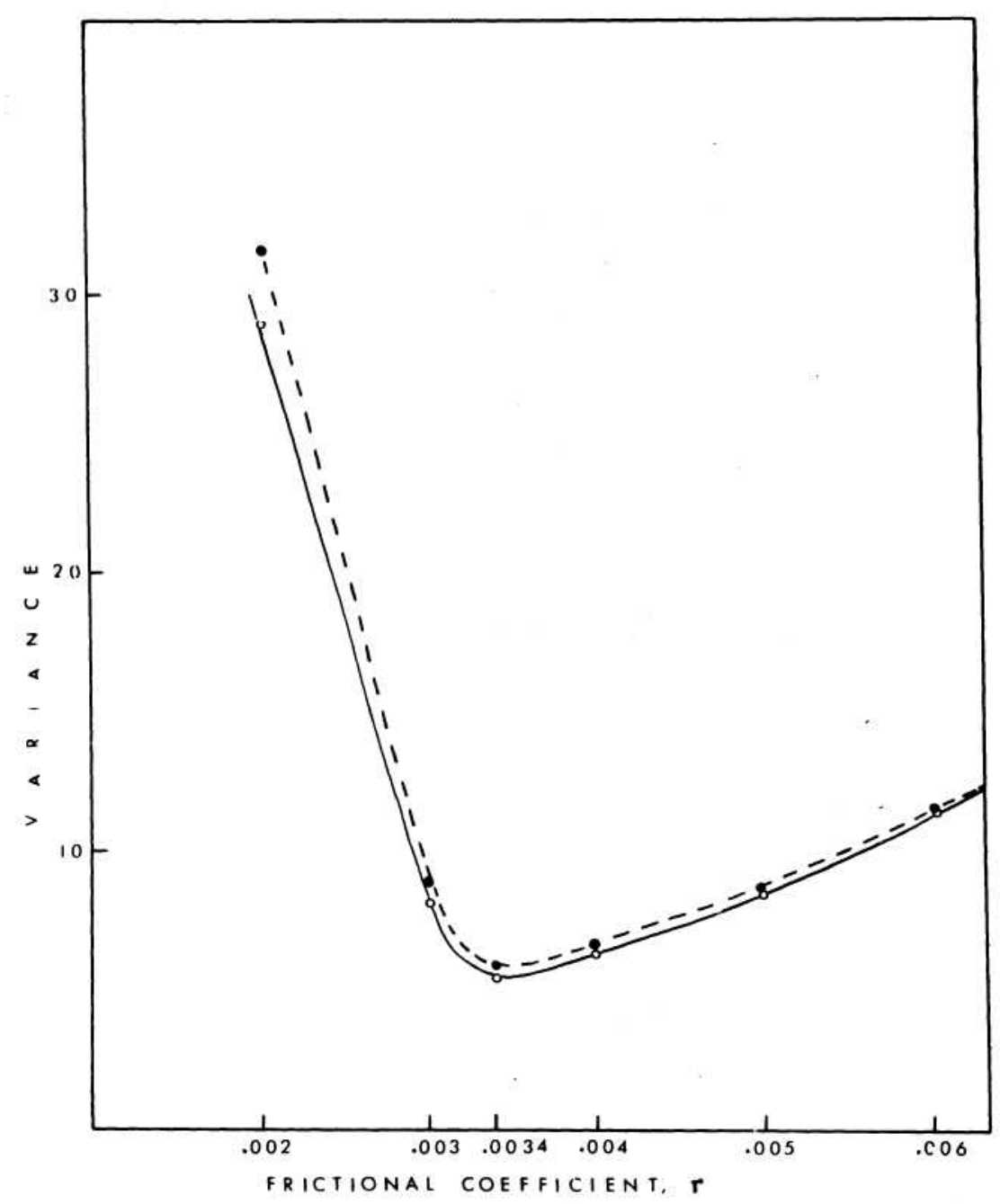

Figure 9. Distribution of average mean .square deviation due to all components over all stations with respect to the frictional coefficient $r$ (the solid line for the consideration of Coriolis force and the dashed line computations without Coriolis force). (Note: the scale of $r$ should be multiplied by $\sqrt{ } \%$ and the curve with Coriolis force should be disregarded - see Foreward). 
One of the advantages of the method considered in this study over Hansen's method is that this method can readily give information on the tidal characteristics of a tidal component. In Hansen's method, the tides for each grid point are computed as a time series, from which one has to perform a harmonic analysis in order to obtain the tidal components. On the otherhand, a disadvantage of this method is its limitation to a system that can be represented by harmonic functions of time.

The numerical method was written in the Fortran IV language for IBM 360/365-G compiler. Storage requirements were $150 \mathrm{~K}$ bytes and machine time approximately 20 minutes for a 5 component calculation with four different values of friction coefficient.

\section{ACKNOWLEDGEMENT}

Primary acknowledgement is extended to Dr. RUSSELL SNYDER for his continuing interest in this study and for his invaluable help in making this work possible. His ideas and views have been most helpful in all phases of investigation.

1 wish to express my sincere appreciation to Dr. CLAES ROOTH for many useful suggestions and comments. His expert comments have always been challenging and stimulating. Equal thanks are due to Dr. Bernard Howard, Dr. Saul Broida, and Mr. Bernard Zetler for the stimulus and encouragement derived from discussions with them.

Special thanks are due to Dr. JEAN FILLOUX for his consistent interest and help in the preparation of the field experiments. It is a pleasure to acknowledge the hospitality of Dr. WILLIAM RICHARDSON for his kind support throughout the study.

I wish to thank Dr. WALTER POWERS and Mr. ROBERT LONG for their considerable assistance. I wish also to express my hearty thanks to all personnel in the Computing Center of the University of Miami for their cooperation.

Finally, an acknowledgement is due to the Administration of International Development (AID) and the National Science Foundation (NSF Grant GA-10153) for support during this study.

\section{REFERENCES}

BICKLEY,W.G. and J. MCNAMEE 1960. Matrix and other direct methods for the solution of systems of Linear Differential Equations. Phil. Roy. Soc. London, A (252): 69-131.

BERGMAN, S. and M.SCHRIFFER 1953. Kernel functions and elliptic differential equations in mathematical physics. Academy Press Inc., New York. 392 pp. 


\section{M. SIDJABAT}

BLAQUIERE, A. 1966. Nonlinear system analysis. Academic Press Inc., New York. $392 \mathrm{pp}$.

Bow DEN, K.P. 1953. Notes on wind drift in a channel in the presence of tidal currents. Proc. Roy. Soc. London A (219): 426 -446.

------- and FAIR BAIRN 1952. Determination of the frictional forces in a tidal current. Proc. Roy. Soc. London A (214) : 371 - 392.

BRETTSCHNEIDER, G. 1967. Anwendungs des Hydrodynamisch-Nemerischen vervahrens zur Ermittlung der M2-mitschwingungsgezeit der Nordsee. Mitt. Inst. Merresk. Hamburg $7: 1-65$.

CARTWRIGHT, D.E. 1968. Unified analysis of tides and surges around North and East Britain. Phil. Trans. Roy. Soc. London A (263): $1-55$.

CHOQUET-BRUHAT 1967. Problems and solutions in mathematical physics. Holdon Day, Inc., San Francisco. 314 pp.

Defant, A. 1919. Untersuchungen uber die Gezeitener-Scheinungen in Mittel-und Randmeeren, in Buchten und Kanalen. Denkschr. Akad. Wiss. Wien. Math. Nat. K1.,S. 96. -- 1961. Physical Oceanography, Vol. II. Mcmillan Co., New York. 593 pp.

DOODSON, A.T. 1956. Tides and storm surges in a long uniform gulf. Proc. Roy. Soc. London A (237): 325 - 343.

------ and H.D.WARBURG, 1941. Admiral manual of tides. H.M.S.O., London. 270 pp.

DRONKERS, J.J. 1962. The linearization of the quadratic resistance term in the equation of motion for a pure harmonic tide in a sea. Proc of the Symposium 1961. Mitt. Inst. Meeresk. Hamburg 1: 195 - 209.

------ 1964. Tidal computation in rivers and coastal waters. Am. Elsevier Now York, $518 \mathrm{pp}$.

FILLOUX, J.H. 1969. Deep sea tide observation, (unpublished manuscript).

FORSYTHE, G.E. and W.R. WASOW, 1960. Finite difference methods for partial differential equations. John Wiley and Sons, New York. 414 pp.

Fox. L. 1950. The numerical solution of elliptic differential equations when the boundary conditions involve a derivative. Phil. Trans. Roy. Soc. London A (24 2): $345-378$. ,

?ALVA, N. 1962. The M2-tide in the English Channel. Proc. of the Symposium 1961. Mitt. Inst. Meeresk. Hamburg 1: $239-243$.

HANSEN, W. 1962. Tides. In: 'The Sea: (M.N. Hill, ed.), Vol. I. John Wiley and Sons, New York: $764-801$.

------ 1962A. Hydrodunamical methods applied to oceanographic problems. Proc. of the Symposium 1961. Mitt. Inst. Meeresk. Hamburg 1:25-34.

HENDERSHOT, M.C. 1966. The numerical integration of Laplace's tidal equation in idealized ocean basin. Mitt. Inst. Meeresk. Hamburg 10: 8- 21.

IPPEN, A.T. 1966. The estuary and coastline hydrodynamics. McGraw Hill, New York. 744 pp.

ISOZAKI,T. and S. UNOKI 1964. The numerical computation of the tsunami in Tokyo Bay caused by the Chilean earthquake in May, 1960. In: "Studies on Oceanography" (Kozo Yoshida, ed.). Univ. of Tokyo Press: $389-402$.

KINSMAN, B. 1965. Wind Waves. Prentice Hall. 670 pp. KREISS, H. 1957. Some remarks about nonlinear oscillation in tidal channel. Tellus 9(1)-53-68

LAMB, H. 1932. Hydrodynamics. Dover Publications, Inc., New York. 738 pp.

MIYASAKI.M. 1964. On boundary conditions to some problems of the long period waves near the coast. In: "Studies of Oceanography" (Kozo Yoshida, ed.). Univ. of Tokyo Press: $383-385$. 
MODELLING OF TIDES IN BASIN

Motz.D.E. 1916. The treatment of singularities of partial differential equation by relaxation methods. Quart. .Appl. Math. (I). ,171-377.

NEUMANN, G. and W.J. PIERSON, JR. 1966. Principle of physical oceanography. Prentice Hall, Englewood Cliffs. 514 pp.

PHOUDMAN.J. 1952. Dynamical Oceanography. Dover Publications, Inc., New York. $109 \mathrm{pp}$.

1960. The conditon that a long period tide shall follow the equilibrium law. Geophys. J. 3(2): $244-249$.

RALSTON, A. and H.S. WILP 1962. Mathematical methods for digital computers. John Wiley and Sons, Inc., New York. 293 pp.

RICHTMYER. R.D. and K.W. MORTON 1967. Difference methods for initial value problems John Wiley and Sons. New York. 405 pp.

Rossiter, J.R. 1962. Long Term Variation in Sea-Level. In: "The Sea" (M.N. Hill, ed.), Vol. I. John Wiley and Sons, New York: $590-610$.

-----1963. Tides, In: Oceanogr Mar. Biol. Ann Her. (H. Barnes, ed.). Ann. Rev. Vol. $1: 11-25$.

SAATY, T.L. and J. BRAM 1964. Nonlinear mathematics. McGraw Hill Co., New York. $381 \mathrm{pp}$.

SGIBNE va, L.A. 1966. Tide calculation in sea of real shape. Oeeanology 6(2): 178 184.

YOUNG, D.M. 1962, The numerical solution of elliptic and parabolic partial differential equations. In: "Survey of numerical analysis" (John Todd, ed.). McGraw Hill Co., New York. 587 pp.

VARGA, R.S. 1962. Matrix iterative analysis. Prentice Hall, Inc., New Jersey. 322 pp 